\title{
Impact of subdominant modes on the interpretation of gravitational-wave signals from heavy binary black hole systems
}

\author{
Feroz H. Shaik, ${ }^{1}$ Jacob Lange, ${ }^{2}$ Scott E. Field, ${ }^{3}$ Richard O’Shaughnessy $\odot,{ }^{2}$ Vijay Varma, ${ }^{4}$ \\ Lawrence E. Kidder $\odot{ }^{5}$ Harald P. Pfeiffer, ${ }^{6}$ and Daniel Wysocki $\odot^{2}$ \\ ${ }^{1}$ Department of Physics, Department of Mathematics, and the Center for Scientific Computing \& \\ Visualization Research, University of Massachusetts Dartmouth, Dartmouth, Massachusetts 02747, USA \\ ${ }^{2}$ Center for Computational Relativity and Gravitation, Rochester Institute of Technology, \\ Rochester, New York 14623, USA \\ ${ }^{3}$ Department of Mathematics and the Center for Scientific Computing \& Visualization Research, \\ University of Massachusetts Dartmouth, Dartmouth, Massachusetts 02747, USA \\ ${ }^{4}$ Theoretical Astrophysics, California Institute of Technology, Pasadena, California 91125, USA \\ ${ }^{5}$ Cornell Center for Astrophysics and Planetary Science, Cornell University, Ithaca, New York 14853, USA \\ ${ }^{6}$ Max Planck Institute for Gravitational Physics (Albert Einstein Institute), \\ Am Mühlenberg 1, 14476 Potsdam, Germany
}

(Received 15 November 2019; accepted 12 June 2020; published 25 June 2020)

\begin{abstract}
Over the past year, a handful of new gravitational wave models have been developed to include multiple harmonic modes thereby enabling for the first time fully Bayesian inference studies including higher modes to be performed. Using one recently developed numerical relativity surrogate model, NRHybSur3dq8, we investigate the importance of higher modes on parameter inference of coalescing massive binary black holes. We focus on examples relevant to the current three-detector network of observatories, with a detector-frame mass set to $120 M_{\odot}$ and with signal amplitude values that are consistent with plausible candidates for the next few observing runs. We show that for such systems the higher mode content will be important for interpreting coalescing binary black holes, reducing systematic bias, and computing properties of the remnant object. Even for comparable-mass binaries and at low signal amplitude, the omission of higher modes can influence posterior probability distributions. We discuss the impact of our results on source population inference and self-consistency tests of general relativity. Our work can be used to better understand asymmetric binary black hole merger events, such as GW190412. Higher modes are critical for such systems, and their omission usually produces substantial parameter biases.
\end{abstract}

DOI: 10.1103/PhysRevD.101.124054

\section{INTRODUCTION}

During their first and second observing runs, the Advanced LIGO [1] and Virgo [2] ground-based gravitational wave $(\mathrm{GW})$ detectors have identified several coalescing compact binaries [3-9]. GW detectors are exceptionally sensitive to very massive objects [10], and the majority of compact binaries observed to date are pairs of $\mathcal{O}\left(30 M_{\odot}\right)$ binary black hole $(\mathrm{BBH})$ systems [9]. The early analysis of these signals used semianalytical approximations to general relativity [11-13]. More recently, better approximations to general relativity have been developed [14-18], which include more of the available physics such as higher-harmonic modes.

Previous investigations have demonstrated that neglecting some of the physics present in real signals produces biased inferences for compact binaries; conversely, including full physics enables sharper inferences. For instance, studies [19-27] have shown that the nonquadrupole modes, while being subdominant, can play a non-negligible role in detection and parameter estimation, particularly for high signal-to-noise-ratio (SNR), large total mass, high mass ratio, or systems favoring an edge-on orientation. In addition, nonquadrupole modes can help break the degeneracy between the binary inclination and distance, which is present for quadrupole-mode-only models (see e.g., [14,28-30]).

The recent observation of GW190412 during the third observing run of LIGO and Virgo has highlighted the significance of higher-harmonic modes for the parameter estimation of unequal mass BBH mergers [31]. Using both precessing and aligned-spin models that included the effects of subdominant modes, it has been demonstrated that a measurable contribution of modes beyond the dominant quadrupolar mode was present in the data of GW190412. This underscores the need for such models for future observing runs.

For the first set of gravitational-wave observations, the massive binary black holes which dominate current observations produce short signals of modest SNRs. For the first event, GW150914 [3], where detailed followups were 
done, the systematic errors due to the quadrupole-modeonly approximation is generally smaller than the statistical errors [32,33], although higher modes may lead to modest changes in some of the extrinsic parameter values [30]. A recent study [32] considering GW150914-like events of near-equal mass and modest amplitude has concluded that neglecting sub-dominant waveform modes did not lead to bias and quadrupole-only models will suffice to characterize the observationally-accessible parameters of astrophysical binary black holes in the immediate future. However, as pointed out in Ref [32], at the time of that study there were no recovery models including higher modes and the systems considered were $q \approx 1.2$ and a detector-frame total mass of 74. Recently, Chatzioannou et al. [34] have reanalyzed GW170729, using IMRPhenomHM [14], SEOBNRv4HM [15], and NRSur7dq2 [18] and found that despite weak evidence for higher-order harmonic modes their inclusion in the analysis leads to increased support for unequal masses.

With newly developed multimode models it is now possible to revisit these questions. We can now compute, for example, the true posteriors using recovery models with multiple harmonic modes that can then be compared to posteriors recovered with dominant modes only. Such comparisons will allow us to precisely quantify the information gained by using subdominant modes. For example, even for an equal-mass system, we observe that the posterior produced without subdominant modes will experience a noticeable shift toward (incorrectly) favoring lighter binary systems with more negative $\chi_{\text {eff }}$ values (cf. Figs. 1 and 4). In fact this preferential bias appears to be a common feature across many of the cases we have considered.

In this paper, we use concrete examples of end-to-end parameter inference to quantify how much approximations that neglect subdominant modes can impact the interpretation of gravitational-wave events. Unlike previous studies, which typically used either a single detector, low signal-to-noise ratios (SNRs), or a Fisher matrix analysis, our fully Bayesian study uses a three-detector network with SNRs typical of detections expected in the near future. We demonstrate these inference biases occur even at moderate signal amplitude for some configurations, growing extreme at amplitudes expected for some sources when LIGO reaches design sensitivity [35].

We also explore additional physics that can be extracted with nonquadropoles modes using a spin-aligned model, such as improved measurability of individual spin components, final mass and spin properties of the remnant, black hole kicks [36], source population inference, and selfconsistency tests of general relativity. For example, in the context of nonspinning BBH systems, Ref. [37] has demonstrated that when higher-modes are omitted from the recovery model, its effect can mimic deviations from general relativity.
Our examples target sources with detector-frame masses $M_{z} \simeq 120 M_{\odot}$, comparable to the detector-frame masses expected for typical near-future binary black hole observations (e.g., pairs of $35 M_{\odot}$ BHs at moderate redshift). For comparison, as ground-based detector networks approach design sensitivity and regularly detect sources near $z \simeq 1$, a merging pair of BHs near the pair-instability mass-gap (50 $M_{\odot}$ ) observed at $z \simeq 1$ would have a detector-frame mass of $M_{z} \simeq 200 M_{\odot}$ [38]. We also consider target sources with mass ratios in the range $1 \leq q \leq 7$. To date most LIGO/Virgo events show support only for systems with mass ratios less than 2 [9]. The recent observation of GW190412 [31] has now shown that we should expect to observe larger mass ratio systems in the future. For example, unequal mass systems are generically expected for $\mathrm{BBH}$ mergers within the accretion disks of active galactic nuclei [39]. Furthermore, the first and second observing runs [9] have already observed compact objects over a mass range of $1.3 M_{\odot}$ to $85 M_{\odot}$ suggesting combinations involving mass-ratios as large as 7 are not unreasonable for LIGO/Virgo to observe.

This paper is organized as follows. In Sec. II we introduce the GW signal model and parameter inference techniques used in this work. In Sec. III we survey the results of parameter inference on a sequence of synthetic high-mass binary black holes with systematically-varied mass ratio, spin, and signal amplitude. We specifically address how higher modes impact inference, comparing parameter inferences performed with the full NRHybSur3dq8 model and with a model truncated to include only $\ell=2$ modes. In $\mathrm{Sec}$. IV we discuss some consequences of our analysis. We conclude in Sec. V with some brief remarks and future work.

\section{PRELIMINARIES}

\section{A. Gravitational wave model}

A coalescing compact binary in a quasicircular orbit can be completely characterized by eight intrinsic parameters, namely the individual masses, $m_{i}$, and spin vectors, $\mathbf{S}_{i}$, of each compact object. Gravitational waveform models and inference codes often employ parametrizations involving the system's total mass, $M=m_{1}+m_{2}$, the mass ratio,

$$
q=m_{1} / m_{2},
$$

where $m_{1} \geq m_{2}$, the dimensionless spins,

$$
\chi_{i}=S_{i} / m_{i}^{2},
$$

on the individual black holes (BHs), and an effective spin parameter [40-42],

$$
\chi_{\text {eff }}=\left(\boldsymbol{S}_{1} / m_{1}+\boldsymbol{S}_{\mathbf{2}} / m_{2}\right) \cdot \hat{L} / M,
$$

which is a weighted combination of the spins projected along the normalized orbital angular momentum vector $\hat{L}$. 
We will express the dimensionless spins in terms of Cartesian components $\chi_{i, x}, \chi_{i, y}, \chi_{i, z}$, expressed relative to the source frame. We define this frame such that the $z$-axis is along the orbital angular momentum direction, which is constant for nonprecessing BBH systems. Since our focus is on the impact of higher-harmonic modes, we restrict ourselves to the 4-dimensional space of nonprecessing $\mathrm{BBHs}$ where nonquadropole, inspiral-merger-ringdown (IMR) models are more mature. Such systems are characterized by $\chi_{i, x}=\chi_{i, y}=0$ and $\left|\chi_{1 z}\right|,\left|\chi_{2 z}\right| \leq 1$.

When discussing waveform models, it is common practice to introduce a complex gravitational-wave strain

$$
\begin{aligned}
& h_{+}\left(t ; t_{c}, l, \phi_{c}, \vec{\lambda}\right)-\mathrm{i} h_{\times}\left(t ; t_{c}, l, \phi_{c}, \vec{\lambda}\right) \\
& \quad=\sum_{\ell=2}^{\infty} \sum_{m=-\ell}^{\ell} h^{\ell m}\left(t-t_{c} ; \vec{\lambda}\right)_{-2} Y_{\ell m}\left(l, \phi_{c},\right),
\end{aligned}
$$

which is subsequently decomposed into a basis of spin-weighted spherical harmonics ${ }_{-2} Y_{\ell m}$. Here $\vec{\lambda} \equiv$ $\left(q, M, \chi_{1 z}, \chi_{2 z}\right)$ is used to denote the signal's dependence on the intrinsic parameters, $l$ is the inclination angle between the orbital angular momentum of the binary and line-of-sight to the detector, $t_{c}$ is the coalescence time, and $\phi_{c}$ is the orbital phase at coalescence. Most gravitational waveform models make predictions for the modes $h^{\ell m}(t)$, from which the gravitational-wave strain detected by a ground-based interferometer,

$$
\begin{aligned}
h(t ; \vec{\Lambda})= & \frac{1}{r} F_{+}(\mathrm{ra}, \operatorname{dec}, \psi) h_{+}\left(t ; t_{c}, l, \phi_{c}, \vec{\lambda}\right) \\
& +\frac{1}{r} F_{\times}(\mathrm{ra}, \operatorname{dec}, \psi) h_{\times}\left(t ; t_{c}, l, \phi_{c}, \vec{\lambda}\right),
\end{aligned}
$$

is readily assembled. The signal's dependence on four additional extrinsic parameters are the polarization angle $(\psi)$, the luminosity distance to the source's center-of-mass $(r)$, and sky location determined by the right ascension (ra) and declination $(\mathrm{dec})$. The antenna patterns $F_{(+, \times)}$project the GW's +- and $\times$-polarization states, $h_{(+, \times)}$, into the detector's frame. We shall use $\vec{\Lambda} \equiv\left(\mathrm{ra}, \operatorname{dec}, \psi, r, t_{c}, l, \phi_{c}, \vec{\lambda}\right)$ to denote the signal's dependence on all 11 parameters defining the problem.

Until recently, all spinning IMR models had set $h^{\ell m}=0$ except for the dominant $h^{2, \pm 2}$ quadrupole modes. The expectation had been that higher modes will not substantially affect parameter inference for the $\mathrm{O} 2$ gravitationalwave observations, which are characterized by low SNRs and mostly face-on events of near-equal mass [22,25,32].

Over the past year or so, three new aligned-spin IMR models have been built to include nonquadropole modes: (i) a phenomenological frequency-domain model, IMRPhenomHM [14], includes the $(\ell,|m|)=(2,2)$, $(3,3),(4,4),(2,1),(3,2),(4,3)$ modes; (ii) an effectiveone-body time-domain model, SEOBNRv4HM [15], includes a similar set of $(\ell,|m|)=(2,2),(3,3),(4,4)$, $(5,5),(2,1)$ modes; (iii) a time-domain surrogate model for hybridized nonprecessing numerical relativity waveforms, NRHybSur3dq8 [16], includes all of the $\ell \leq 4$ and $(5,5)$ spin-weighted spherical harmonic modes but not the $(4,1)$ or $(4,0)$ modes.

Our study will use NRHybSur3dq8 as it both includes the most modes and is expected to be more accurate when evaluated within its training region (cf. Fig 6 from Ref. [16]) of mass ratio $q \leq 8$, and $\left|\chi_{1 z}\right|,\left|\chi_{2 z}\right| \leq 0.8$. For the $20 \mathrm{~Hz}$ starting frequency considered here, this model is valid for the entire LIGO band for stellar mass binaries with total masses as low as $2.25 M_{\odot}$. We evaluate the model through the PYTHON package GWSurrogate ${ }^{1}[44,45]$. The GWSurrogate package provides direct access to the GW's harmonic modes $h^{\ell m}(t)$ appearing in the sum (4).

By comparing to NR, Ref. [16] has computed the NRHybSur3dq8 model's mismatches (averaged over many points on the sky) as a function of total mass using the Advanced LIGO design sensitivity noise curve. For the $120 M_{\odot}$ total mass systems predominantly used in our studies, the single-detector mismatches have a median value of $1 \times 10^{-5}$. A sufficient condition for two waveform models (in this case NR and NRHybSur3dq8) to be considered indistinguishable is [32,46-48]

$$
\mathcal{M}<\frac{D}{2 \rho^{2}}
$$

where $\mathcal{M}$ is the mismatch and $\rho$ is the signal-to-noise ratio (SNR). Here $D$ is an unknown constant that is sometimes associated with the number of model parameters [49], with $D=4$ for our spinning BBH model. Furthermore, if the likelihood can be approximated by a Gaussian then an expression for $D$ can be obtained in terms of a chi-squared distribution with 4 degrees of freedom [50]. Using this value for $D$ and a typical mismatch value quoted above, we find that the NRHybSur3dq8 model will give robust parameter estimates so long as $\rho \lesssim 450$. Even using pessimistic values $(D=1$ and the 95th percentile of mismatch errors $7 \times 10^{-5}$ ) we find that NR and our model will be indistinguishable according to Eq. (6) so long as $\rho \lesssim 85$.

For context, we note that in the first and second observing runs most BBH signals had a network SNR of about 15 and spanning a range of 10 to 30. In the upcoming observing run we would expect typical $\mathrm{BBH}$ SNRs to be between 10 and roughly 40, based on the cumulative distribution of the loudest SNR $\rho$ among $n$ identified events $\left(\left[1-(\rho / 10)^{3}\right]^{n}\right.$ using a fiducial value $n=30$ ). We caution the reader that in practice the

\footnotetext{
${ }^{1}$ We use GWsurrogate version $0.9 .\{4,5\}$, which exactly agrees with the lalsimulation [43] implementation of the NRHybSur3dq8 model.
} 
condition in Eq. (6) should only be taken as a rough estimate. For instance, it features an unknown constant $D$ while the NR waveforms themselves have small, systematic sources of error that would prevent any model to claim indistinguishability from general relativity beyond estimates of this systematic error [51]. Finally, the definition of "indistinguishable" is not synonymous with "identical posterior distributions". Indeed, Fig. 4 shows that even for simple systems at low SNR, which easily satisfy Eq. (6), there can be noticeable discrepancies between the recovered posteriors. For example, using a single interferometer the mismatch between $\ell_{\max }=5$ and $\ell_{\max }=2$ models for a nonspinning, equal-mass system is 0.0021 , and so Eq. (6) is satisfied at SNRs less than 30.

Due to the absence of higher-mode models for spinning $\mathrm{BBH}$ systems until recently, previous parameter-inference studies that have focused on the information content available higher modes have either used quadrupole-only (recovery) models or leveraged the Fisher matrix framework. For high-accuracy, high-SNR scenarios involving the 3 -detector network neither of these are fully sufficient. For example, with the quadrupole-only model the reference ("true") posterior will not be possible to compute in principle. Additionally, some of these models may have modeling errors in the dominant mode that could become noticeable at high SNR [52-54].

\section{B. Bayesian inference}

The likelihood of GW data in Gaussian noise has the form (up to normalization),

$$
\begin{aligned}
\ln \mathcal{L}(\lambda, \theta)= & -\frac{1}{2} \sum_{k}\left\langle h_{k}(\lambda, \theta)-d_{k} \mid h_{k}(\lambda, \theta)-d_{k}\right\rangle_{k} \\
& -\left\langle d_{k} \mid d_{k}\right\rangle_{k},
\end{aligned}
$$

where $h_{k}$ are the predicted response of the $k^{\text {th }}$ detector due to a source with parameters $(\lambda, \theta)$ and $d_{k}$ are the detector data in the $k^{\text {th }}$ instrument; $\lambda$ denotes the combination of redshifted total mass $M_{z}$ and the remaining intrinsic parameters needed to uniquely specify the binary's dynamics; $\theta$ represents the seven extrinsic parameters (4 spacetime coordinates for the coalescence event and 3 Euler angles for the binary's orientation relative to the Earth); and $\langle a \mid b\rangle_{k} \equiv \int_{-\infty}^{\infty} 2 d f \tilde{a}(f)^{*} \tilde{b}(f) / S_{h, k}(|f|)$ is an inner product implied by the $k^{\text {th }}$ detector's noise power spectral density (PSD) $S_{h, k}(f)$. In practice we adopt both low- and highfrequency cutoffs $f_{\max }, f_{\min }$ so all inner products are modified to

$$
\langle a \mid b\rangle_{k} \equiv 2 \int_{|f|>f_{\min },|f|<f_{\max }} d f \frac{[\tilde{a}(f)]^{*} \tilde{b}(f)}{S_{h, k}(|f|)} .
$$

The joint posterior probability of $\lambda, \theta$ follows from Bayes' theorem:

$$
p_{\text {post }}(\lambda, \theta)=\frac{\mathcal{L}(\lambda, \theta) p(\theta) p(\lambda)}{\int d \lambda d \theta \mathcal{L}(\lambda, \theta) p(\lambda) p(\theta)},
$$

where $p(\theta)$ and $p(\lambda)$ are priors on the (independent) variables $\theta, \lambda$. Following most previous work $[9,55,56]$, we adopt uninformed separable priors for parameter inference.

\section{RIFT}

To construct the posterior distribution, we use the RIFT algorithm [56], which iteratively constructs and refines an approximation to the marginal likelihood

$$
\mathcal{L}_{\text {marg }} \equiv \int \mathcal{L}(\lambda, \theta) p(\theta) d \theta
$$

which appears in Bayes' theorem for the marginal posterior distribution for $\lambda$. We use an existing program (ILE, which Integrates the Likelihood over Extrinsic parameters) to perform the necessary marginalization, for each fixed source [33,57-59], by marginalizing the likelihood of the data over the seven parameters characterizing the spacetime coordinates and orientation of the binary relative to the earth; see $[57,60]$ paper for technical details.

To achieve rapid turnaround times, we use the new GPUaccelerated implementation of ILE [60]. Working on the CARNiE cluster, which includes 15 NVIDIA Tesla V100 GPU-enabled nodes, our current configuration completes each of the binary black hole analyses presented in this work in about 15 to 20 hours. When using all 15 GPUs, a single ILE step for an SNR $=30$ case takes about 1 hour to finish.

Following the RIFT algorithm [56], we iteratively construct an approximation to the likelihood by generating and drawing from approximate posterior distributions, until our posterior distribution converges. At each iteration, the likelihood is approximated using Gaussian process regression with a squared-exponential kernel, with hyperparameters tuned to the likelihood evaluations available at that iteration.

\section{INTRINSIC-PARAMETER BIASES}

In this section, we present parameter estimation (PE) results from sources listed in Table I. All synthetic datasets use PSDs generated from data near GW170814 [6], when all three detectors were operational, and are created with zero noise realizations. Specifically the synthetic detector data is exactly equal to the expected response due to our $\mathrm{GW}$ source. Since detector noise is assumed to be colored Gaussian noise with zero mean, using zero noise with the likelihood defined in Eq. (7) makes our analysis equivalent to an average over an ensemble of analyses which use infinitely many noise realizations [32]. For all runs, $f_{\text {min }}$ and $f_{\max }$ from Eq. (8) are $20 \mathrm{~Hz}$ and $2000 \mathrm{~Hz}$, respectively. 
TABLE I. Parameters of synthetic sources: This table shows the parameters of all the synthetic sources used in this paper. $l$ is the inclination angle between the line of sight of the observer and the total angular momentum vector, $q$ is the mass ratio defined with $q>1$ [see Eq. (1)], $M$ is the detector-frame total mass, and $\chi_{*}$ are the components of the normalized spins [see Eq. (2)]. As we use a nonprecessing model, we set all of the in-plane spin components to 0 . All luminosity distances are set such that the network signal-tonoise ratio achieves the value specified under the SNR column. For example, in our $q=7$ sequence the most extreme values of spin, $\chi_{\text {eff }}=-0.8$ and $\chi_{\text {eff }}=0.8$, are located at $181.4720 \mathrm{Mpc}$ and $452.5185 \mathrm{Mpc}$, respectively. This large discrepancy in distance is due to the orbital hangup effect and is explained in greater detail in Fig. 11. Other extrinsic parameters are fixed to the following values: right ascension is $\mathrm{RA}=0.0$, declination is $\mathrm{DEC}=1.5707963$, and the polarization angle is $\psi=\pi / 4$.

\begin{tabular}{lcccccr}
\hline \hline ID\# & $\imath$ & $q$ & $M\left(M_{\odot}\right)$ & $\chi_{1 \mathrm{z}}$ & $\chi_{2 \mathrm{z}}$ & \multicolumn{1}{c}{$\mathrm{SNR}$} \\
\hline 1 & $\pi / 4$ & 2.267 & 127.1 & 0.72 & 0.0 & 30 \\
2 & $3 \pi / 4$ & 1.00 & 120.0 & -0.80 & -0.80 & 30 \\
3 & $3 \pi / 4$ & 1.00 & 120.0 & -0.50 & -0.50 & 30 \\
4 & $3 \pi / 4$ & 1.00 & 120.0 & 0.0 & 0.0 & $10,30,70$ \\
5 & $3 \pi / 4$ & 1.00 & 120.0 & 0.50 & 0.50 & 30 \\
6 & $3 \pi / 4$ & 1.00 & 120.0 & 0.80 & 0.80 & 30 \\
7 & $3 \pi / 4$ & 4.00 & 120.0 & -0.8 & -0.8 & 30 \\
8 & $3 \pi / 4$ & 4.00 & 120.0 & -0.5 & -0.5 & $10,30,70$ \\
9 & $3 \pi / 4$ & 4.00 & 120.0 & 0.0 & 0.0 & 30 \\
10 & $3 \pi / 4$ & 4.00 & 120.0 & 0.5 & 0.5 & 30 \\
11 & $3 \pi / 4$ & 4.00 & 120.0 & 0.8 & 0.8 & 30 \\
12 & $3 \pi / 4$ & 7.00 & 120.0 & -0.8 & -0.8 & 30 \\
13 & $3 \pi / 4$ & 7.00 & 120.0 & -0.5 & -0.5 & 30 \\
14 & $3 \pi / 4$ & 7.00 & 120.0 & 0.0 & 0.0 & 30 \\
15 & $3 \pi / 4$ & 7.00 & 120.0 & 0.5 & 0.5 & 30 \\
16 & $3 \pi / 4$ & 7.00 & 120.0 & 0.8 & 0.8 & 30 \\
\hline \hline
\end{tabular}

Each synthetic dataset includes an injected signal from the expected response at each detector due to our GW source using the NRHybSur3dq8 model and including all of the surrogate's available $\ell_{\max }=5$ modes (see Sec. II A for the exact modes, which, for example, only includes $(5,5)$ among the $\ell=5$ modes). The model generates a waveform such that the instantaneous initial frequency of the $(2,2)$ mode has a frequency of $8 \mathrm{~Hz}$, which ensures the $(5,5)$ mode's instantaneous initial frequency is out-of-band. We taper the beginning and end portions of the waveform to avoid artificial oscillations in the Fourier domain. In particular, since NR waveforms (and therefore the NRHybSur3dq8 model) do not go to zero by the end of the simulation, we have found it necessary to taper the last portion of the ringdown signal.

We adopt conventional mass and distance priors, uniform in detector-frame mass and in the cube of the luminosity distance. For our nonprecessing spins, we adopt a uniform prior for $\chi_{i, z} \in[-0.9,0.9]$. Section IVA considers the effect of using an alternative spin prior in the context of high SNR events.

Each of the following subsections describe a set of related runs, varying one of the problems' parameters at a time. For each source configuration, we present parameter estimates recovered using all of the available higher modes $\ell_{\max }=5$ (we may sometimes refer to this as the "true" or reference posterior) and compare with posteriors recovered using the same model restricted to only the $\ell_{\max }=2$ modes (using $|m|=\{2,1\})$. In Secs. III A $(q=1)$, III B $(q=4)$, and III C $(q=7)$ we vary the spin configurations of $\chi_{1 \mathrm{z}}=$ $\chi_{2 \mathrm{z}}=\{-0.8,-0.5,0.0,0.5,0.8\}$ while keeping the network SNR fixed at $30 .^{2}$ For this sequence of runs, our choice of inclination angle, $l=3 \pi / 4$, is neither face-on nor edge-on, but rather constitutes a "general" configuration. In Sec. III D we consider varying the SNR to explore its effect on marginalized posterior distributions.

It is known that the contribution of subdominant modes toward the signal's power increases as the inclination angle is increased from a face-on $(l=0)$ to an edge-on $(l=\pi / 2)$ configuration. As such, we expect our observed biases to be larger (smaller) when compared to a face-on (edge-on) system at the same network SNR value. This general expectation was recently confirmed by Kalaghatgi et al. [61], where the importance of subdominant modes for nonspinning systems was quantified by systematically varying the inclination angle across a range of values. In our study we have instead fixed the inclination angle to a value typical of an $\mathrm{O} 2$ event [9] while systematically exploring the impact due to spin. As such our results are complementary to those of Ref. [61].

\section{A. $q=1$}

We first look at a set of equal mass runs with the different spin configurations mentioned above. It is well known that the relative power of subdominant harmonic modes are minimized for equal mass $\mathrm{BBH}$ systems, so these cases are expected to minimize bias. Previous studies $[19,22,25,32]$ have either found negligible bias (for faceon systems), small bias (for edge-on systems), or quoted results averaged over the source orientation where again only very small biases were found. At the time of these studies $[19,22,25,32]$, however, there were no recovery models for near-equal mass spinning BBH systems including subdominant modes so these results were only suggestive. Here we confirm the general expectation of smaller bias at $q=1$, while also making more precise the nature of the bias by comparing the true posterior to the approximate one found with $\ell_{\max }=2$ modes only. For example, in all cases the true posterior's peak is located at $q=1$, while some of the biased posteriors have a non-negligible offset often peaking closer to $q \sim 1.25$. From Fig. 1 we also

\footnotetext{
${ }^{2}$ Given a fixed starting frequency, systems with their $\mathrm{BH}$ component spins (anti-)aligned with the orbital angular momentum will be (shorter) longer. As a result, to achieve a fixed SNR the spin (anti-)aligned systems must be place located (closer) farther as compared to a reference nonspinning system.
} 

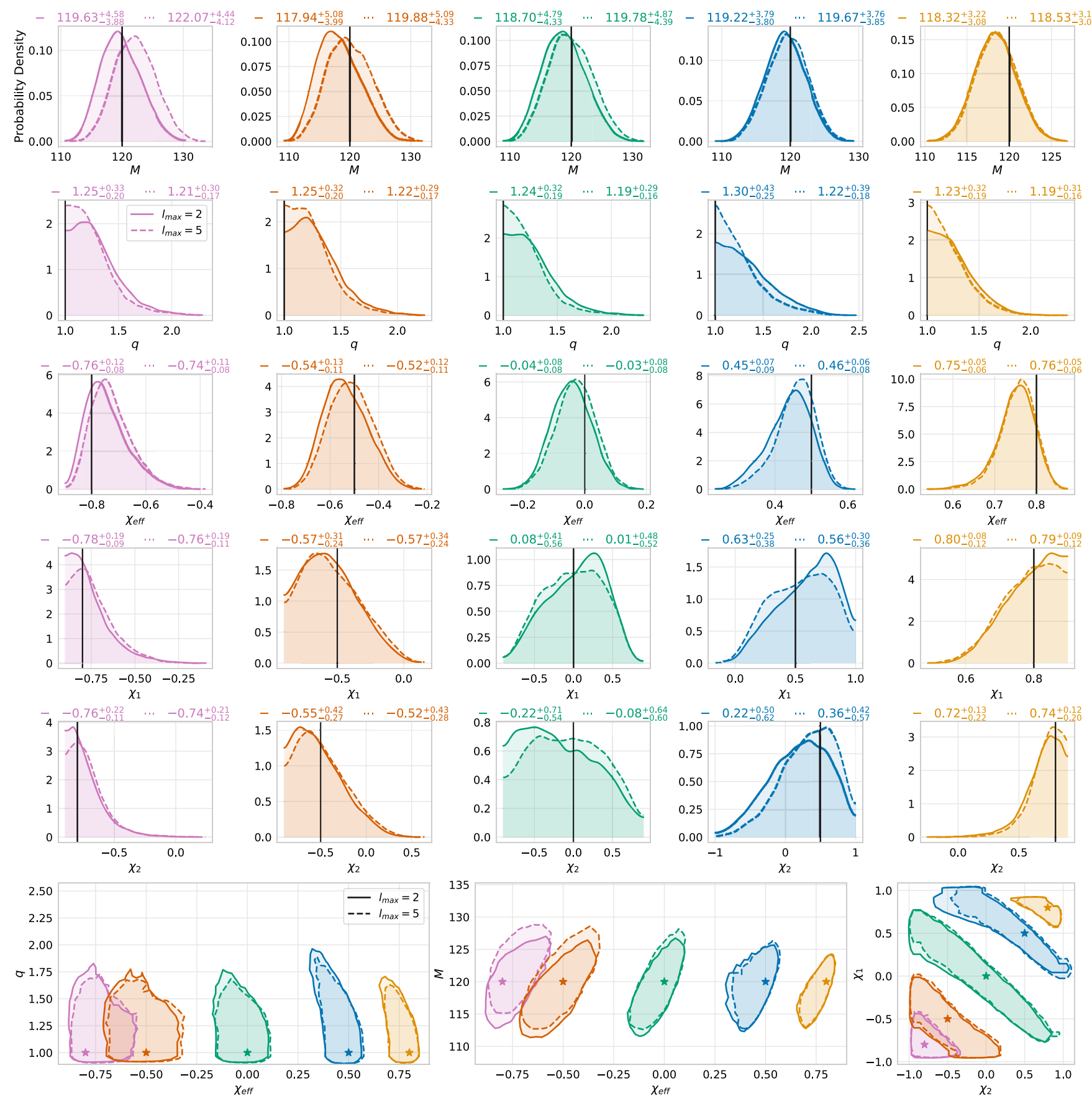

FIG. 1. Non-HM and HM runs for $q=1$ spin set, with SNR $=30$ and $M=120$ : The first five rows show the $M, q, \chi_{\text {eff }}, \chi_{1 z}, \chi_{2 z}$ onedimensional marginal distributions, where among this set of figures each column corresponds to a different synthetic source recovered with either all $\ell_{\max }=5$ modes (dashed line) or $\ell_{\max }=2$ modes (solid line). Our figures are organized such that the injected spin is systematically increased from left to right, where the synthetic source runs are ID2 $\left(\chi_{\text {eff }}=-.8\right)$, ID3 $\left(\chi_{\text {eff }}=-.5\right)$, ID4 $\left(\chi_{\text {eff }}=0\right)$, ID5 $\left(\chi_{\mathrm{eff}}=.5\right)$, and ID6 $\left(\chi_{\mathrm{eff}}=.8\right)$. In each figure's title, we report the median value and the $90 \%$ confidence intervals of the marginalized $1 \mathrm{D}$ distribution for the $\ell_{\max }=2$ (left) and $\ell_{\max }=5$ (right) cases. A solid black vertical line denotes the true parameter value. The final bottom row corresponds to the joint distributions for $q$ vs $\chi_{\text {eff }}$, M vs $\chi_{\text {eff }}$, and $\chi_{1, z}$ vs $\chi_{2, z}$ for all five injections.

observe noticeable shifts in the posteriors $90 \%$ confidence region for anti-aligned configurations.

Figure 1 shows the posterior distributions of the intrinsic parameters for all the different spin configurations. The solid lines represent runs that were done with $\ell_{\max }=2$ modes, and the dashed lines represent runs that include all available $\ell_{\max }=5$. For each run, there is some degree of difference between the $\ell_{\max }=2$ and $\ell_{\max }=5$ runs. As anticipated by Ref. [19], which used a non-Bayesian approach and a single detector, this discrepancy between 
the two distributions become more extreme as the spins increase toward negative spin. For example, for negative spins there are noticeable shifts in the $M$ vs $\chi_{\text {eff }}$ posteriors. We emphasize that even for the simplest case (equal mass and zero spin), differences between the two results are visible. Although parameter recovery is not biased in the sense that all of the injection values lie within their $90 \%$ confidence regions, it is also clear from the figure that the median recovered using all subdominant modes is almost always closer to the injection value. This is contrary to the general expectation that subdominant modes are largely irrelevant for equal-mass systems [22,25,32]. Section III D explores how different network SNRs affect the bias for these systems; Appendix follows up on the curious differences seen in the simplest case of zero spin, equal mass.

\section{B. $q=4$}

We next increase our set of sources to $q=4$, a configuration that is most relevant to GW190412-like events. Similar to the $q=1$ case, as far as we are aware, the existing literature for parameter estimation is comprised of results for non-spinning recovery models [22], results for near-equal mass without multi-mode recovery models [32], or Fisher matrix-based studies $[19,25]$. None of those studies consider the 3-detector network configuration and a multimodal recovery model with fully Bayesian inference. At larger mass ratios, our study confirms the general expectations described in Ref. [19], although the observed bias is often even larger than expected; compare to the typical errors indicated by corresponding green, red, and blue curves in Fig. 6 of Ref. [19] for our fiducial mass. We also are able to more carefully quantify the nature of the bias by comparing to the true posteriors. In particular, similar to the $q=1$ systems just considered, neglecting subdominant modes consistently shifts the posterior toward more extreme antialigned spin configurations with lighter total mass.

Figure 2 shows the posterior distributions for $\chi_{\text {eff }}$ vs $q$ and $\chi_{\text {eff }}$ vs $M$ for all the different spin configurations. The solid lines again represent runs that were done with $\ell_{\max }=$ 2 modes, and the dashed lines represent runs that include all available $\ell_{\max }=5$. Similar to Sec. III A, we again see that the differences become more extreme as the spins increase toward negative spin. Comparing the same spin configures between $q=1$ and $q=4$ runs, it is clear that increasing the mass ratio dramatically increases the bias between the non$\mathrm{HM}$ and HM runs. In particular, there are now many cases where parameter estimates recovered with $\ell_{\max }=2$ modes do not lie within their $90 \%$ confidence regions. Looking at the two-dimensional posteriors, for example, shows many cases where either the $\ell_{\max }=2$ posterior either does not contain the injection value or it is noticeably shifted from the true posterior. By comparison, in almost all of the $\ell_{\max }=5$ cases, the marginal posteriors almost perfectly peak at the true parameters. One notable exception is the $\chi_{\text {eff }}=-0.8$ case (the purple distributions in Fig. 2) where the true parameters seem to lie just inside the $90 \%$ confidence region. We suspect this is due to a combination of (i) the injection being very close to the boundaries of the prior and (ii) the posterior for a $\chi_{\text {eff }}=-0.8$ injection is much wider than the corresponding $\chi_{\text {eff }}=0.8$ value, which does not show this unexpected behavior.

$$
\text { C. } q=7
$$

Finally, we analyze sources with $q=7$. Figure 3 shows the posterior distributions of the intrinsic parameters for all the different spin configurations. The solid lines again represent runs that were done with $\ell_{\max }=2$ modes, and the dashed lines represent runs that include all available $\ell_{\max }=5$. As expected and consistent with the trend seen in the previous two subsections, we see substantial biases are often introduced in $M, q$ and $\chi_{\text {eff }}$ if higher modes are omitted, especially for systems with large negative spin. Only the higher-mode model is able to make reliable parameter estimates, except for the large, positive spin configurations where a quadrupole-only model continues to do reasonably well. In some cases the biased posterior does not even overlap with the true one, which would be problematic for likelihood-reweighting techniques [62], which require similar posterior distributions.

Somewhat unexpectedly, however, is that the $\chi_{1}=\chi_{2}=$ 0.8 system's posterior shows almost no effect from neglecting subdominant modes; any effect that is present is smaller than the corresponding equal-mass system with $\chi_{1}=\chi_{2}=$ -0.8 . We believe this can be explained by the orbital hangup effect [63], whereby given two otherwise identical systems the one with larger aligned spin will experience more orbits before merger. Consequently, the $\chi_{1}=\chi_{2}=$ 0.8 configuration will have more in-band cycles, and subdominant modes are known to be suppressed during the inspiral phase. We briefly elaborate on this effect in the conclusions.

\section{Effect of network SNR on biases}

In the previous subsections, it was shown that a significant bias exists at $\mathrm{SNR}=30$, even for the simplest systems. This subsection is dedicated to investigating how the SNR affects the bias. Here we use all the different SNR runs from ID4 and ID8 in Table I. Figures 4 and 5 show the posterior distributions for ID4 and ID8 respectively. As the SNR increases, the posteriors become more precise for both the non-HM and HM results (i.e., the statistical errors get smaller). However, the HM results converge on the true parameters while the non-HM results converge to a point offset from the true parameter (i.e., the systematic errors remain the same size and will dominate the statistical uncertainties). As GW detectors get more sensitive, the need for HM will become paramount even for the simplest of events. More sensitive detectors will potentially bring into view more exotic configurations at low SNRs which can also be problematic. For example, the weakest 

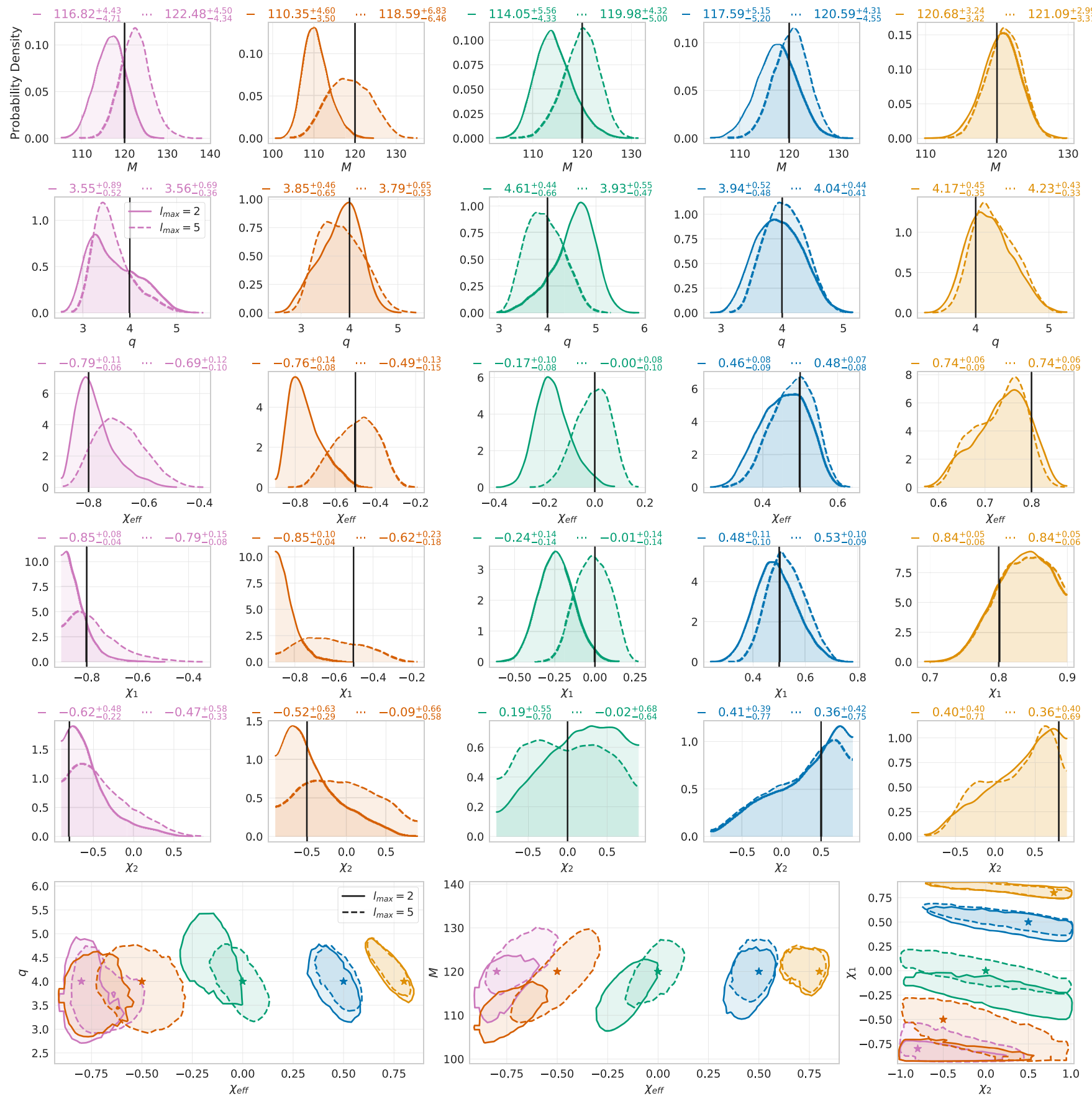

FIG. 2. Non-HM and HM runs for $q=4$ spin set, with $\mathrm{SNR}=30$ and $M=120$ : The first five rows show the $M, q, \chi_{\mathrm{eff}}, \chi_{1 z}, \chi_{2 z}$ onedimensional marginal distributions, where among this set of figures each column corresponds to a different synthetic source recovered with either all $\ell_{\max }=5$ modes (dashed line) or $\ell_{\max }=2$ modes (solid line). Our figures are organized such that the injected spin is systematically increased from left to right, where the synthetic source runs are ID7 $\left(\chi_{\text {eff }}=-.8\right)$, ID8 $\left(\chi_{\text {eff }}=-.5\right)$, ID9 $\left(\chi_{\text {eff }}=0\right)$, ID10 $\left(\chi_{\mathrm{eff}}=.5\right)$, and ID11 $\left(\chi_{\mathrm{eff}}=.8\right)$. In each figure's title, we report the median value and the $90 \%$ confidence intervals of the marginalized $1 \mathrm{D}$ distribution for the $\ell_{\max }=2$ (left) and $\ell_{\max }=5$ (right) cases. A solid black vertical line denotes the true parameter value. The final bottom row corresponds to the joint distributions for $q$ vs $\chi_{\text {eff }}, \mathrm{M}$ vs $\chi_{\text {eff }}$, and $\chi_{1, z}$ vs $\chi_{2, z}$ for all five injections.

$q=4, \chi_{\text {eff }}=-0.5$ system has noticeable bias. This could be anticipated by noting that the mismatch between $\ell_{\max }=5$ and $\ell_{\max }=2$ models at this injection value is 0.06989 and so Eq. (6) is not satisfied.
One particularly challenging configuration was the loudest $q=4, \chi_{\text {eff }}=-0.5$ system shown in Fig. 5 (solid blue). In particular, the posterior recovered with the $\ell_{\max }=2$ model shows evidence for a secondary peak widely 

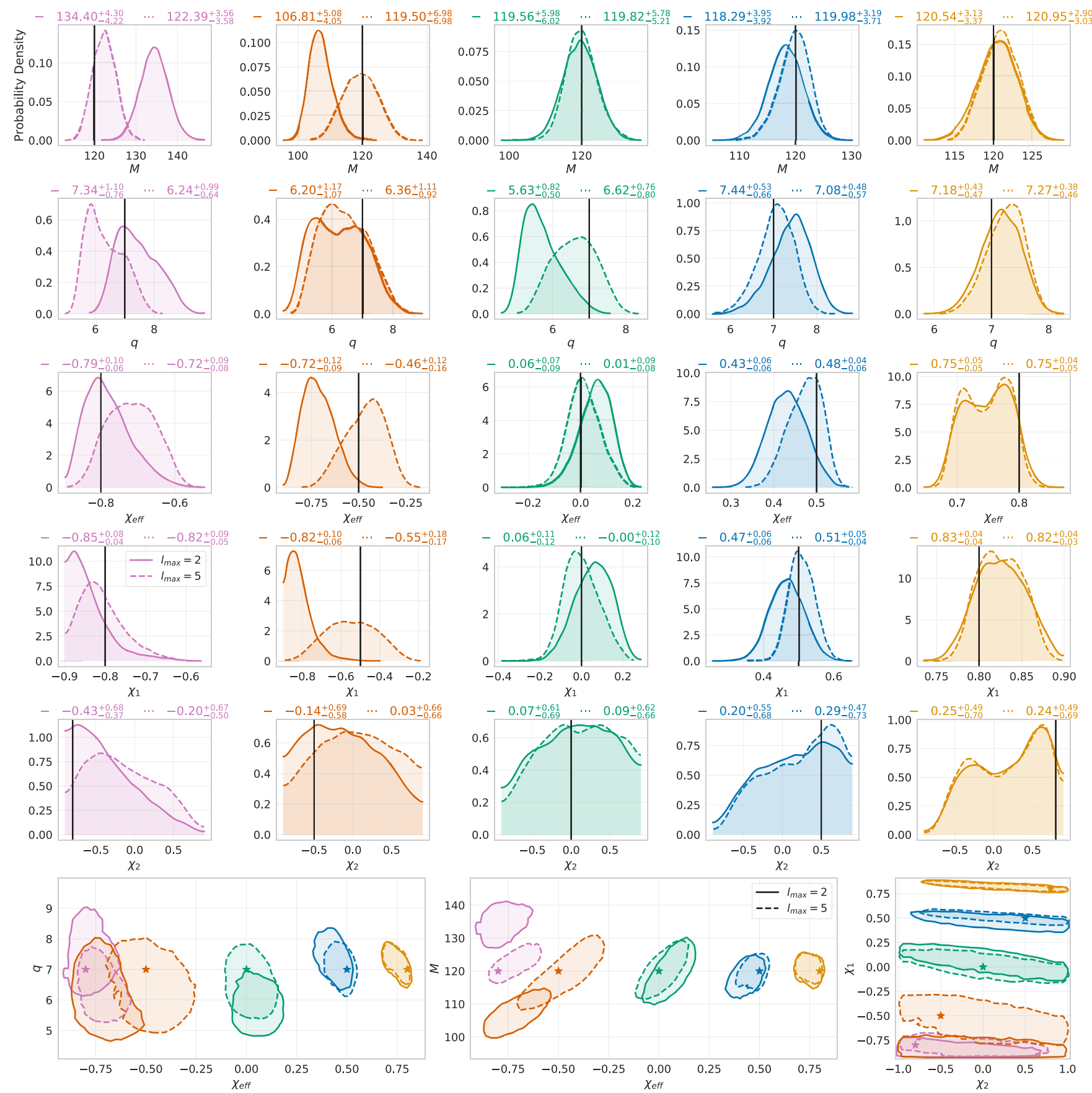

FIG. 3. Non-HM and HM runs for $q=7$ spin set, with SNR $=30$ and $M=120$ : The first five rows show the $M, q, \chi_{\text {eff }}, \chi_{1 z}, \chi_{2 z}$ onedimensional marginal distributions, where among this set of figures each column corresponds to a different synthetic source recovered with either all $\ell_{\max }=5$ modes (dashed line) or $\ell_{\max }=2$ modes (solid line). Our figures are organized such that the injected spin is systematically increased from left to right, where the synthetic source runs are ID12 ( $\left.\chi_{\text {eff }}=-.8\right)$, ID13 $\left(\chi_{\text {eff }}=-.5\right)$, ID14 $\left(\chi_{\text {eff }}=0\right)$, ID15 $\left(\chi_{\text {eff }}=.5\right)$, and ID16 $\left(\chi_{\text {eff }}=.8\right)$. In each figure's title, we report the median value and the $90 \%$ confidence intervals of the marginalized 1D distribution for the $\ell_{\max }=2$ (left) and $\ell_{\max }=5$ (right) cases. A solid black vertical line denotes the true parameter value. The final bottom row corresponds to the joint distributions for $q$ vs $\chi_{\text {eff }}, \mathrm{M}$ vs $\chi_{\text {eff }}$, and $\chi_{1, z}$ vs $\chi_{2, z}$ for all five injections.

separated from the primary one. We checked this unexpected feature by directly comparing the values of the likelihood in a small neighborhood around both peaks. The presence of these two widely separated peaks proved to be challenging for the current implementation of the ILE/RIFT algorithm, which uses a single interpolant of the loglikelihood surface. As a result, running this case took a significantly longer time while also achieving a comparatively lower accuracy, where the accuracy is quantified by the effective number of adaptive Monte Carlo samples. 

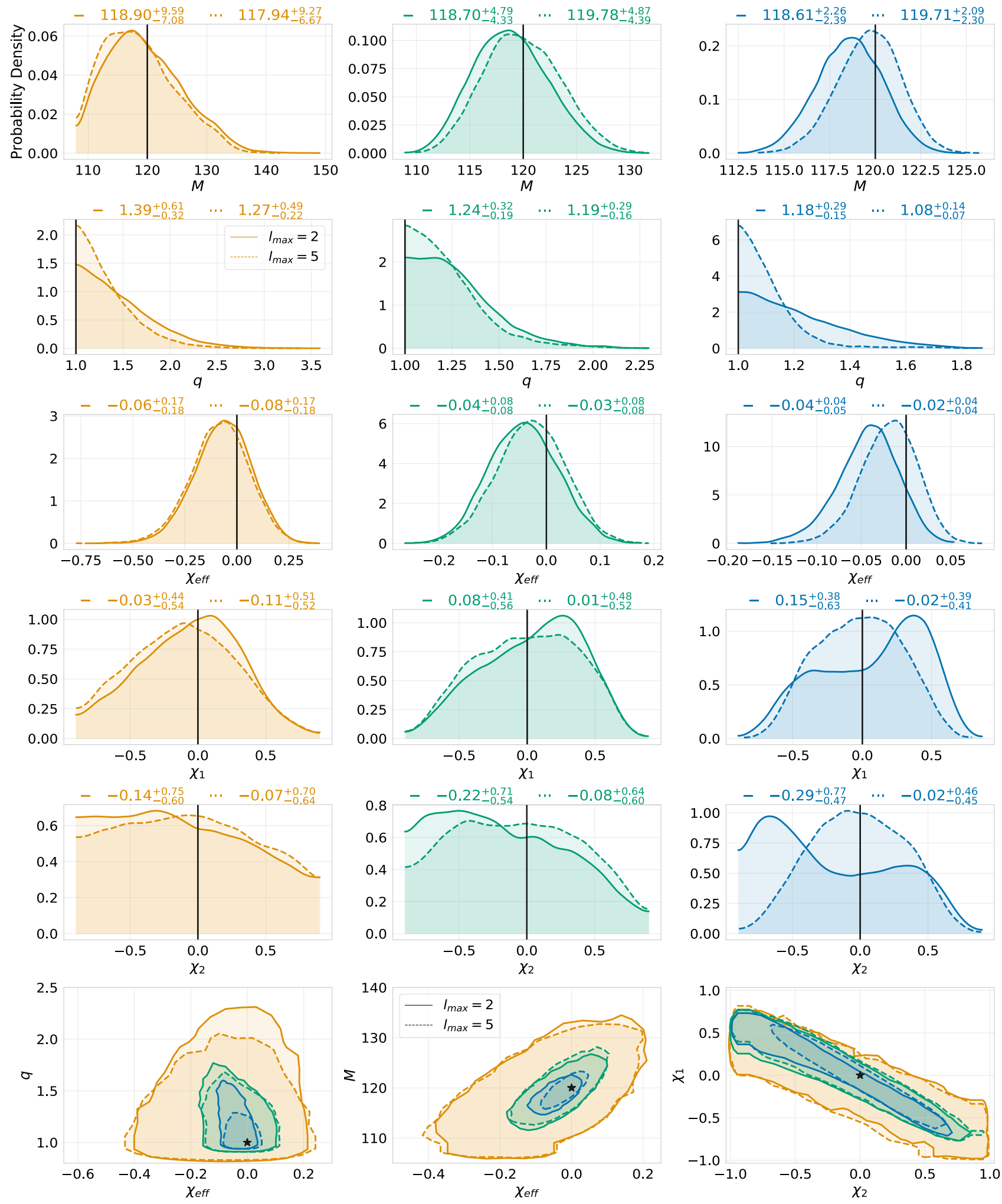

FIG. 4. Non-HM and HM runs for a $q=1, M=120$, and zero-spin source (ID4), for different SNRs: The first five rows show the $M$, $q, \chi_{\text {eff }}, \chi_{1 z}, \chi_{2 z}$ one-dimensional marginal distributions, where among this set of figures each column corresponds to a different synthetic source recovered with either all $\ell_{\max }=5$ modes (dashed line) or $\ell_{\max }=2$ modes (solid line). Our figures are organized such that the signal's network SNR is systematically varied as 10 (orange), 30 (green), and 70 (blue), corresponding to the left, middle, and right columns, respectively. A solid black vertical line denotes the true parameter value. The final bottom row corresponds to the joint distributions for $q$ vs $\chi_{\text {eff }}$, M vs $\chi_{\text {eff }}$, and $\chi_{1, z}$ vs $\chi_{2, z}$ for all three injections. 

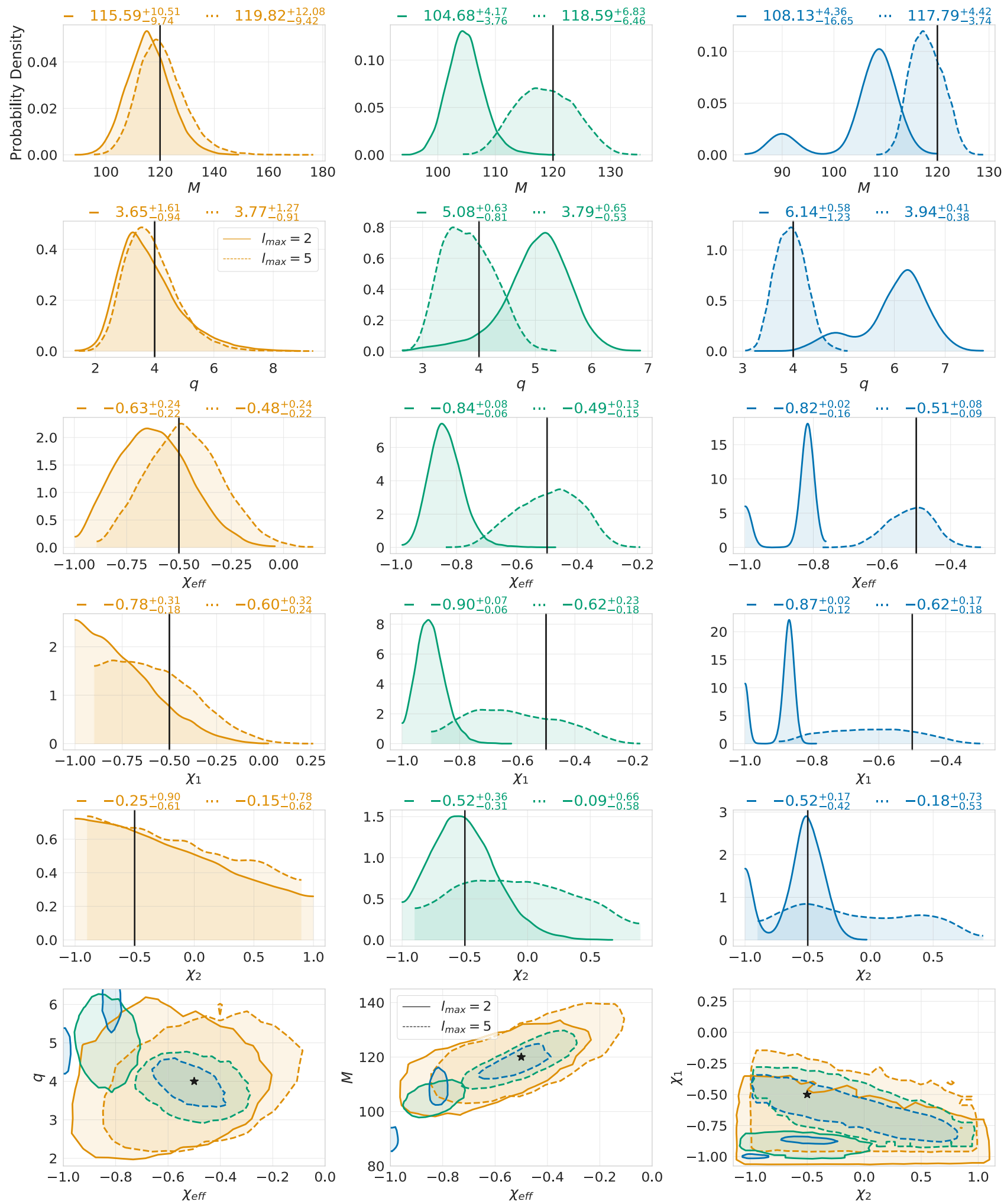

FIG. 5. Non-HM and HM runs for a $q=4, M=120$, and $\chi_{\text {eff }}=-0.5$ source (ID8), for different SNRs: The first five rows show the $M, q, \chi_{\text {eff }}, \chi_{1 z}, \chi_{2 z}$ one-dimensional marginal distributions, where among this set of figures each column corresponds to a different synthetic source recovered with either all $\ell_{\max }=5$ modes (dashed line) or $\ell_{\max }=2$ modes (solid line). Our figures are organized such that the signal's network SNR is systematically varied as 10 (orange), 30 (green), and 70 (blue), corresponding to the left, middle, and right columns, respectively. A solid black vertical line denotes the true parameter value. The final bottom row corresponds to the joint distributions for $q$ vs $\chi_{\text {eff }}, \mathrm{M}$ vs $\chi_{\text {eff }}$, and $\chi_{1, z}$ vs $\chi_{2, z}$ for all three injections. 

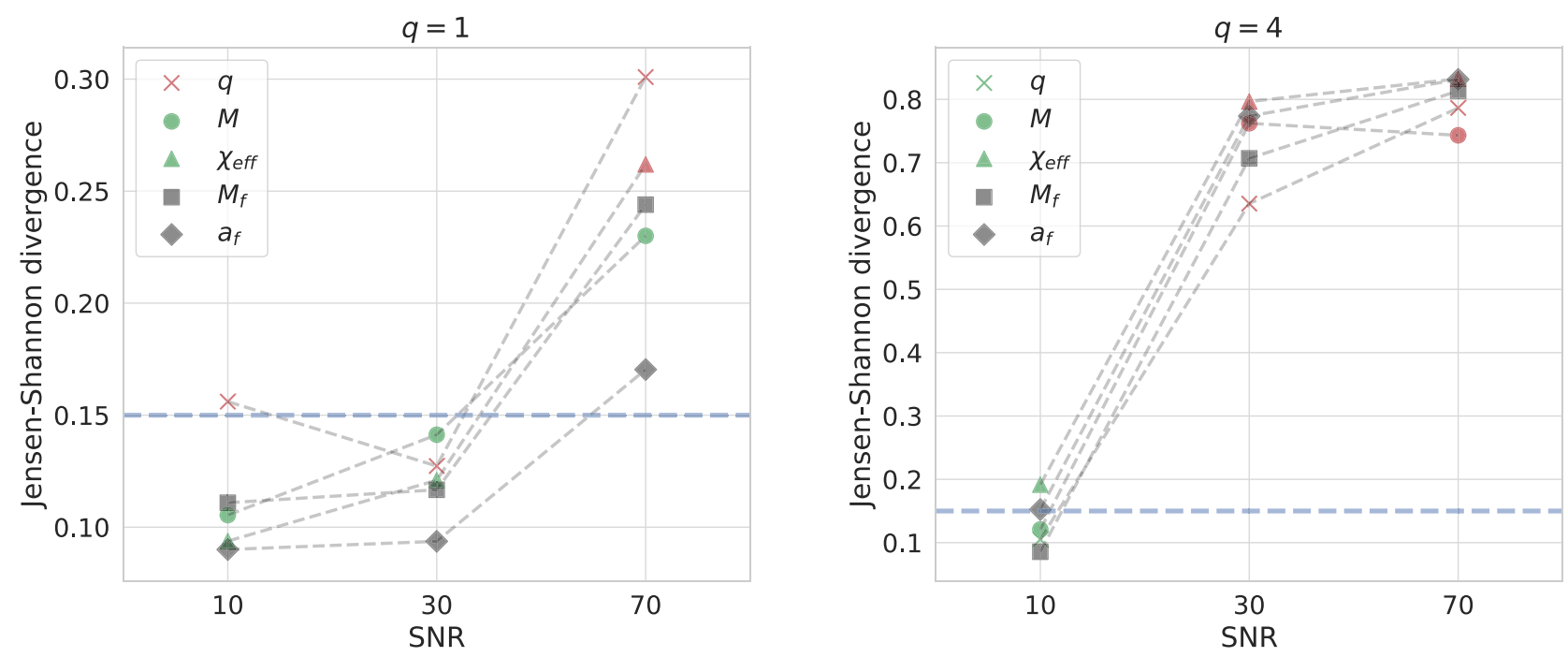

FIG. 6. The importance of higher modes for loud signals: bias vs SNR: These panels show the JSD vs SNR for source ID4 (left panel) and ID8 (right panel). Different markers indicate which one-dimensional marginal distribution was used to evaluate the JSD, which are depicted in Figs. (4) and (5) for ID4 and ID8, respectively. The dashed horizontal blue line demarcates a commonly used threshold for unacceptably large bias. Markers colored in red indicate that the true value falls outside the $90 \%$ credible interval region for the $\ell_{\text {max }}=2$ case (significant bias in the recovered parameter value), while those colored in green indicate the opposite. For $\ell_{\max }=5$, the true value is almost always within the $90 \%$ credible interval region except the parameter $q$ in the $q=1$ case, where the true value lies at the edge; despite not being in the he $90 \%$ credible interval the marginalized distribution for $q$ obtains its maximum value at $q=1$ (cf. row 2 of Fig. 4). Markers in gray indicate the JSD for the final remnant masses and spins.

This case underscores that for high SNR events the omission of subdominant modes can introduce highly complex likelihood surfaces, and prove challenging to explore accurately. Within the RIFT framework, a recently implemented Gaussian mixture model sampler is expected to more efficiently sample from complicated likelihood surfaces. This case also demonstrates how incorrect models can accidentally yield good recovery of some parameters: the marginalized posterior for $\chi_{2}$ (solid blue curve) looks remarkably accurate around the primary peak despite the joint posterior (bottom right panel) being nowhere near the true value.

To quantify the bias between the non-HM and HM runs, we consider two commonly used measures of bias: (i) classifying the recovery of a particular parameter as biased if the injected parameter value is outside of the $90 \%$ confidence region and (ii) the Jensen-Shannon divergence (JSD) between the different parameter distributions. Given two probability distributions $p(x)$ and $g(x)$, the JSD is defined as

$$
D_{\mathrm{JS}}(p \mid g)=\frac{1}{2}\left(D_{\mathrm{KL}}(p \mid s)+D_{\mathrm{KL}}(g \mid s)\right)
$$

where $s=1 / 2(p+g)$ and

$$
D_{\mathrm{KL}}(p \mid g)=\int p(x) \log _{2}\left(\frac{p(x)}{g(x)}\right) \mathrm{d} x,
$$

is the Kullback-Leibler divergence (KLD) between the distributions $p$ and $g$, measured in bits. For context, this is the same calculation the LVC performed in [9] to quantify the agreement between different models. When measured in bits, the JSD is bounded below by 0 . For a sense of scale, the KL divergence between two one-dimensional Gaussians with identical standard deviations but differing means $\mu_{1}, \mu_{2}$ is $\left(\mu_{1}-\mu_{2}\right)^{2} / 2 \sigma^{2} \ln 2$; inverting, JSD $=0.2$ corresponds to $\mu_{1}-\mu_{2} \simeq 0.5 \sigma$.

Figure 6 shows the JSD vs SNR and the simple "bias classifier" for both the ID4 and ID8 runs, respectively. Following the discussion in the LSC's recently published gravitational-wave transient catalog [9] (cf. Appendix 2.B), we consider two marginalized posteriors to be sufficiently different (i.e., biased) if the JSD is greater than $\approx 0.15$. This number corresponds to a $\mathrm{SNR} \simeq 30$ for non-spinning, equal-mass binaries; $\mathrm{SNR} \simeq 10$ at $q=4$ and $\chi_{1 \mathrm{z}}=\chi_{2 \mathrm{z}}=$ -0.5 . Since subdominant modes become more important at larger mass ratios and more negative values of $\chi_{\text {eff }}$, the quoted SNRs provide convenient lower bounds for similar systems. For example, we expect HMs will also affect the posterior for systems with SNRs $\geq 30$ and $q>1, \chi_{\text {eff }} \leq 0$ (similar to ID4); for systems with SNRs $\geq 10$ and $q>4$, $\chi_{\text {eff }} \leq-0.5$ (similar to ID8).

\section{DISCUSSION}

\section{A. Effect of different spin priors}

Besides the impact of subdominant modes, the ability to accurately measure the spin parameters is also influenced by the choice in spin prior [64], which is not well informed by astrophysical observations or source population models. 
In our study, we have used a prior which is uniform in $\chi_{\mathrm{z}}$ (P1). However, many of the LVC's analysis assume a prior that is uniform in spin magnitude, $|\vec{\chi}|$, and on the 2-sphere, which, for our nonprecessing model, would induce a prior by projection of $\vec{\chi}$ along the orbital angular momentum vector (P2). When assuming this spin prior, the peak of the $\mathrm{PDF}$ of the individual component spins will strongly favor zero. To see how these two significantly different priors affect the ability to measure the spins, we compare posteriors for two runs ID2 and ID6 with $\mathrm{SNR}=30$ assuming the two different priors. Figure 7 shows the individual $\chi_{* z}$ spins as well as the effective spin $\chi_{\text {eff }}$ for each spin prior. Despite using a strong source, all spin parameters are significantly perturbed by the prior choice, similar to results found in previous work [64].

\section{B. Consequences of biases for remnant properties and consistency tests}

Using the posterior distributions of the BBH system's component masses and spins one can compute the remnant mass, $M_{f}$, and spin, $a_{f}$ of the final (merged) black hole. The values of $\left(M_{f}, a_{f}\right)$ are interesting in their own right as they can be used to infer a population of astrophysical compact objects that formed through the merger of a $\mathrm{BBH}$ system. Another use of $\left(M_{f}, a_{f}\right)$ is to test the consistency of general relativity by predicting these remnant values found from (i) the post-merger portion of the signal which is described by a ringdown signal characterized entirely by $\left(M_{f}, a_{f}\right)$ and (ii) the inspiral portion of the signal where we compute the BBH system's component values and, using numerical relativity, arrive at an alternative estimate of $\left(M_{f}, a_{f}\right)$. If general relativity correctly describes the system's entire evolution, we should expect the remnant values found through each to be mutually consistent [65]. A closely related test uses the remnant values computed with the inspiral-only portion of the signal to infer the expected quasinormal mode (QNM) of ringdown signal, and then comparing this predicted QNM spectrum with the QNMs estimated directly from the ringdown-only portion of the data [65]. A different, but related, set of tests of the no-hair theorem also benefit from the inclusion of both higher harmonics and as well as quasinormal mode overtones [66].

All of these studies require accurate measurement of the system's remnant masses and spins. For example, unacceptably large bias in these quantities could provide misleading evidence for failed GR consistency tests, unless the quadrupole-only premerger and postmerger models make a serendipitously incorrect inference of the remnant properties (i.e., both models are incorrect but in a consistent manner).

In this subsection we explore bias in the remnant properties implied by the posterior distributions computed in Sec. III as the SNR increases. We compute the remnant mass and spin magnitude by evaluating the high-accuracy fitting formula provided by the surfinBH PYTHON package
[67] on the posteriors computed using $\ell_{\max }=5$ and $\ell_{\max }=2$ recovery waveform models.

As the first example, where we expect minimal bias, we consider the $q=1$, zero-spin source system (ID4) whose posterior distributions for SNRs $=\{10,30,70\}$ are reported in Fig. 4 from which we compute remnant posteriors in Fig. 8 (left set of figures). While the true remnant values are contained within all of the joint posteriors's $90 \%$ credible region, we begin to see modest bias indicating impact from the higher-modes when the signal's strength reaches an SNR value of 70. This is quantified in Fig. 6 which shows the Jensen-Shannon divergence for $M_{f}$ and $a_{f}$ are 0.24 and 0.17 , respectively. For context, values above 0.15 are typically considered to reflect non-negligible bias [9]. At all values of the SNR, we find the $\ell_{\max }=5$ posterior more tightly constrains the true values.

Figure 8 also shows a similar sequence for the $q=4$, $\chi_{\text {eff }}=-0.5$ source (ID8) where now the true remnant values are no longer contained within the $90 \%$ credible intervals by $\mathrm{SNR}=30$. As seen from Fig. 6, the JS divergence is already close to, or greater than, 0.15 at $\mathrm{SNR}=10$. This suggests that higher modes are very important when estimating the remnant values from such systems, and neglecting them would incorrectly lead to a failure of the IMR consistency test for essentially any event we might conceivably observe similar to ID8.

\section{Consequences of biases on population reconstruction}

In a second and more qualitative example of the impact of parameter biases due to neglect of physics, we consider astrophysical inference for the mass, mass ratio and spin distribution of coalescing BHs. For example, consider an $\mathrm{SNR}=30$, zero-spin BBH event with $q=4$. As illustrated by the green curves in Fig. 2, inferences which neglect HMs would deduce negative effective spin (and a more extreme mass ratio). A single source with definitively negative $\chi_{\text {eff }}$ would be interpreted as a strong indication for dynamical formation in samples of less than several hundred mergers. Such biased inferences for high-amplitude sources could thus be misinterpreted to support qualitatively different formation channels (e.g., dynamical formation) than supported by the true parameters, which are well characterized by multimodal PE.

More typically, parameter biases due to model incompleteness enter more insidiously into astrophysical inference, since population inference relies on combining information from multiple sources and since systematic biases impact all sources at a similar level. Following [68], we estimate that parameter biases $\Delta x=x^{\text {true }}-x^{\text {median }}$ will be significant for a population of $N$ sources if the bias can be identified in the population mean by stacking observations: in other words, if $\Delta x \gtrsim \sqrt{\sigma_{\text {stat }}^{2}+\sigma_{\text {astro }}^{2}} / \sqrt{N}$ where $\sigma_{\text {stat }}$ and $\sigma_{\text {astro }}$ are the statistical error in $x$ and the width of 

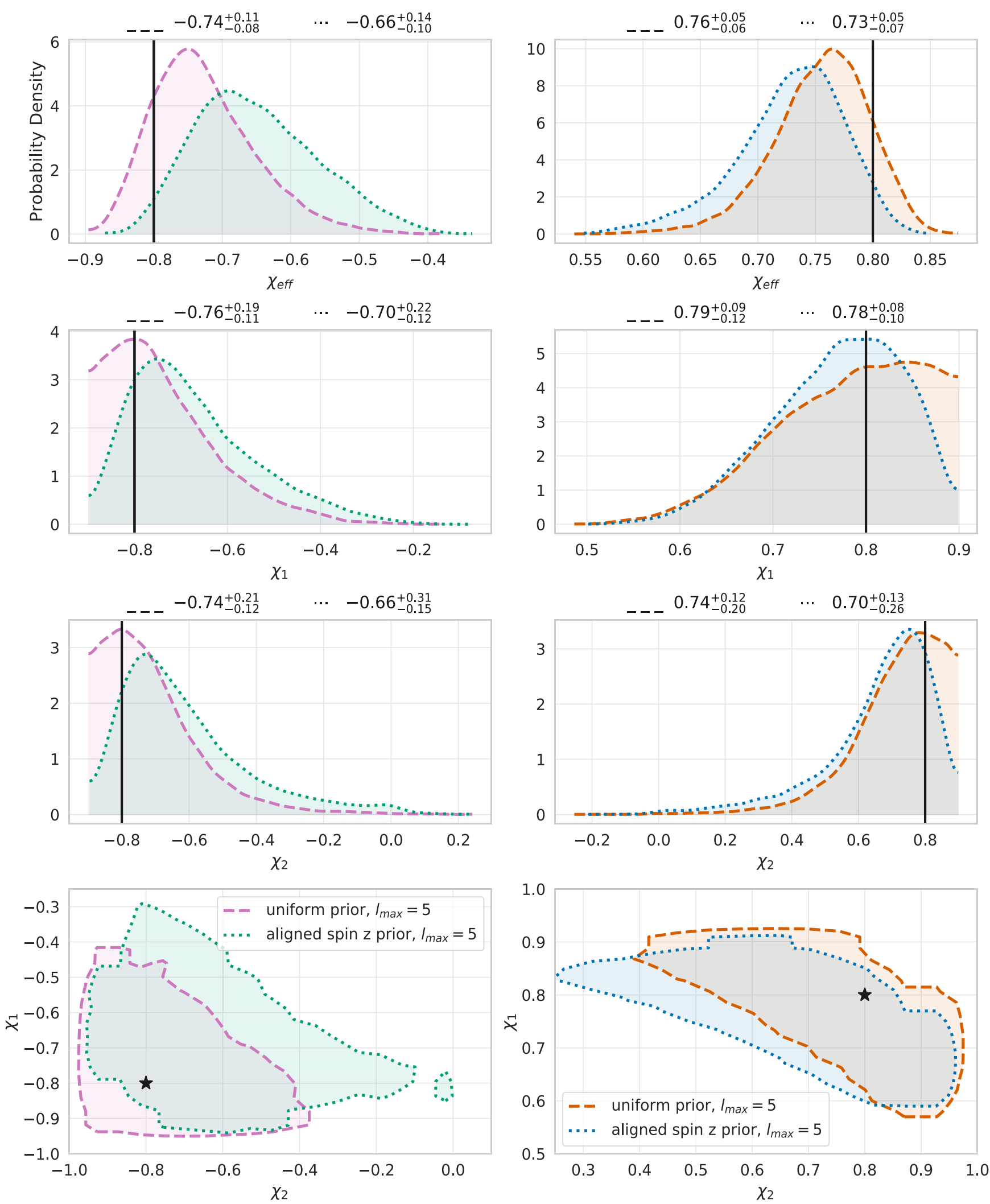

FIG. 7. The effect of priors on spin measurability: Individual and effective spin parameter recovery assuming two different priors, using synthetic datasets ID2 $\left(q=1, \chi_{\text {eff }}=-.8\right)$ and ID6 $\left(q=1, \chi_{\text {eff }}=.8\right)$ with SNR $=30$. The dashed curve represents the results using a prior that assumes uniform spin magnitudes in $\chi_{z}(\mathrm{P} 1$; uniform prior), and the dotted curve represents the results using a prior that assumes uniform spin magnitudes in $\vec{\chi}$ (P2; aligned spin z prior). Despite the high value of SNR used here, we observe that the choice of prior has a significant influence on the recovered posteriors. 

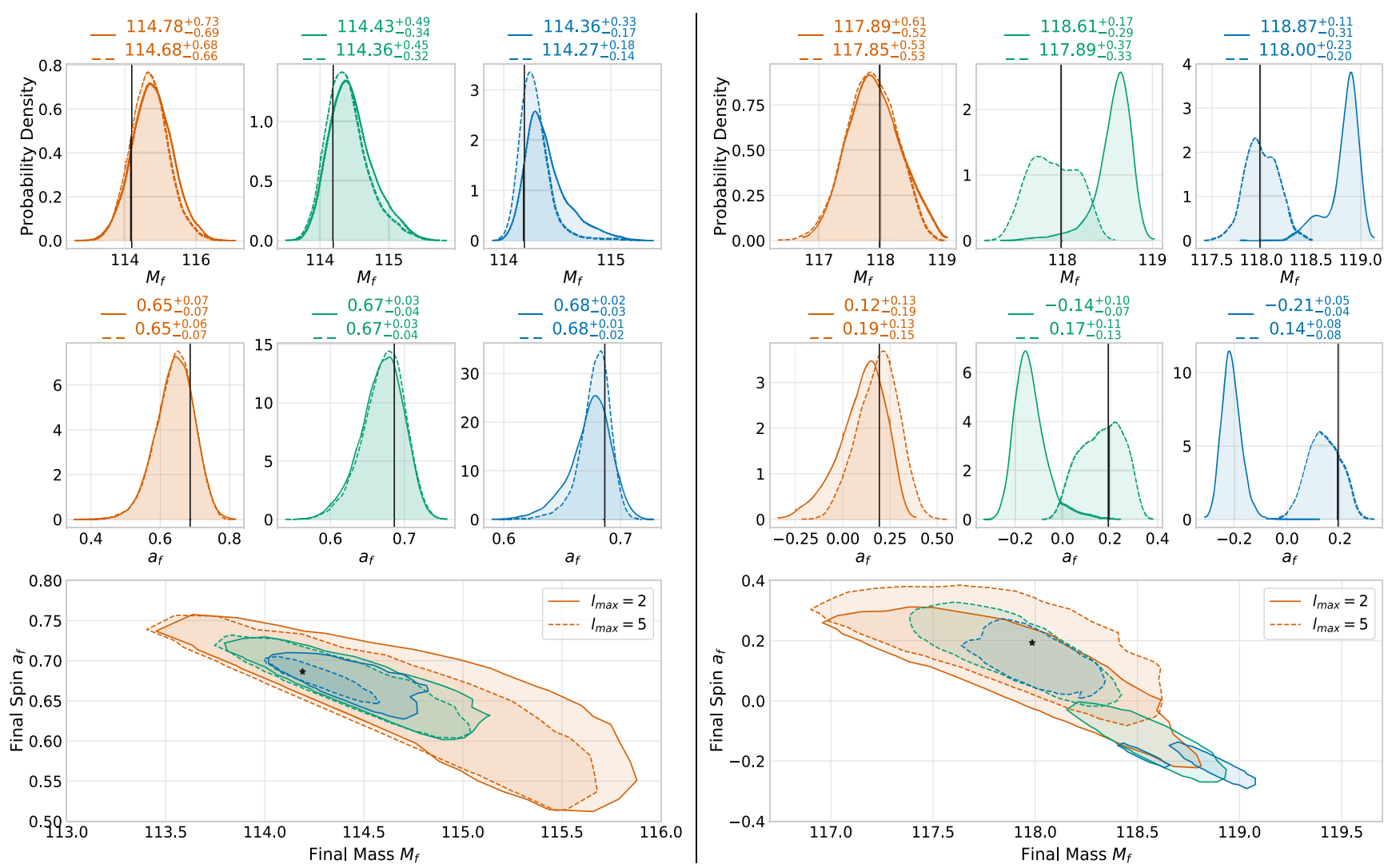

FIG. 8. Effect of higher-order modes on remnant values and IMR consistency tests: These panels show marginal distributions for remnant properties of the redshifted mass, $M_{f}$, and spin, $a_{f}$, for a non-spinning, $q=1$ source (ID4; left panels) and $\chi_{\text {eff }}=-0.5, q=4$ source (ID8; right panels). Our figures are organized such that the signal's network SNR is systematically varied as 10 (orange), 30 (green), and 70 (blue), corresponding to the left, middle, and right columns of each panel.

the astrophysical distribution of $x$, respectively. In terms of the JSD we anticipate that systematic differences in waveforms must produce a change in posteriors less than JSD = $0.15 / N$ to have no effect on population inference. Our examples show that even for zero-spin (but unequal-mass) binaries, inferences about the mass ratio, total mass, and effective spin in moderate-SNR sources can be significantly biased by the lack of HM. If a population of unequal massratio binaries exist and has a spin distribution qualitatively similar to the seemingly low-spin BH population identified in $\mathrm{O} 2$, even inferences drawn from a handful of observations could be noticeably biased about BH masses and spins.

\section{A GW170729-like source}

While much of our focus has been on fiducial BBH systems, it is also interesting to consider sources that are similar to events from the most recent observing run. In this subsection, we analyze a synthetic source that has parameters (cf. ID1 in Table I) similar to GW170729, one of the more interesting events from $\mathrm{O} 2$. As mentioned in [9,61], the SNR of GW170729 was 12. However, to better highlight the importance of HMs for this event, we instead consider a GW170729-like event located at a distance such that the SNR is 30. For consistency with other synthetic events analyzed throughout this paper, we set $\chi_{\text {eff }}=0.5$ as its true value, which is near the upper end of the $90 \%$ credible interval $\mathrm{t}$ [34]. Note that although $\chi_{\text {eff }}=0.5$, we now have $\chi_{1 z} \neq \chi_{2 z}=0$. We continue using a uniform spin magnitude in $\chi_{\mathrm{z}}$ as our spin prior.

Figure 9 shows the posterior distributions for the runs that include only $\ell_{\max }=2$ (solid lines) and include all the $\ell_{\max }=5$ (dashed lines). As with all the results in Sec. III, we see a significant bias between the two runs in all the parameters. For example, we see that the $\ell_{\max }=5$ model does a much better job at recovering the individual spin components as well as placing somewhat tighter constraints on the spin of the larger $\mathrm{BH}, \chi_{1 z}$. Interestingly, we see a similar shift in $q$ and $\chi_{\text {eff }}$ that was observed in a recent reanalysis of the actual GW170729 event [34]. As our detectors continue to get more sensitive, we will increasingly see events with parameter and SNR values similar to the synthetic source ID1 considered here.

\section{E. Comparison to previous works}

Previous studies [19-27,30,32,61] have also considered the impact of subdominant modes on parameter estimation, 

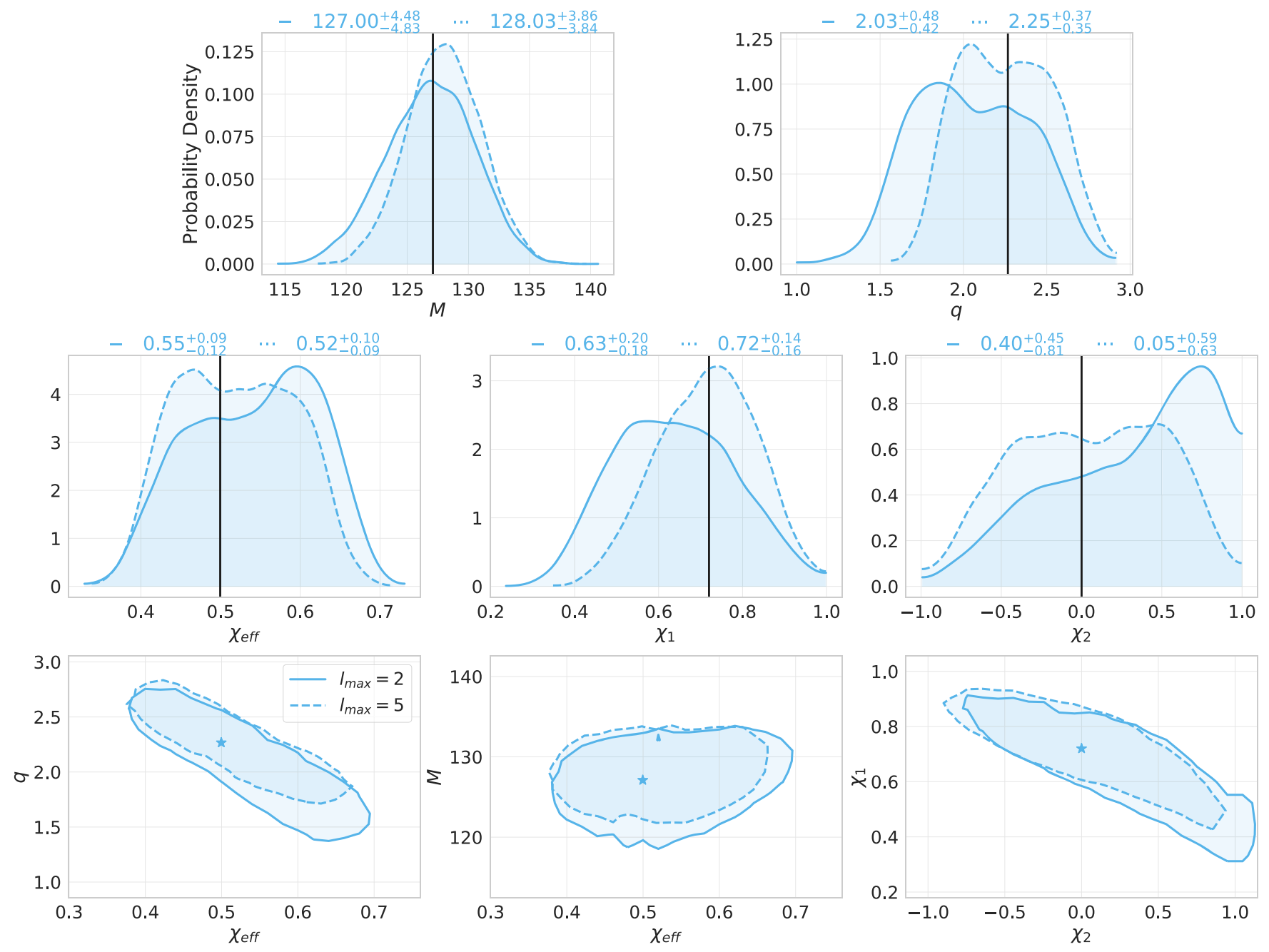

FIG. 9. GW170729-like event: Posterior plots for the ID1 run: $q=2.267, M\left(M_{\odot}\right)=127.1, \chi_{1 z}=0.72, \chi_{2 z}=0.0$, SNR $=30$. The solid and dashed lines represent the $\ell_{\max }=2$ and $\ell_{\max }=5$ runs respectively. When including HM, we are able to improve the recovery of individual spin components. We also see a significant shift in the $q$ and $\chi_{\text {eff }}$ distributions.

and we have made qualitative comparisons to some of these works throughout our paper.

In this subsection, we furnish a more quantitative comparison by considering one commonly used measure of bias. Instead of using the Jensen-Shannon divergence to compare two marginalized posterior distributions, we now compute the bias,

$$
\beta_{\lambda}=\frac{\Delta \lambda}{\sigma_{\lambda}}
$$

as a ratio of the systematic error, $\Delta \lambda=\left|\lambda_{\text {injected }}-\lambda_{\text {recovered }}\right|$, to the $1 \sigma$ statistical error in the one-dimensional posterior, $\sigma_{\lambda}$. The quantity $\beta_{\lambda}$ can be used to compare with Varma et al. [19,25] and Kalaghatgi et al. [61]. We follow the choice of Refs. [22] where $\lambda_{\text {recovered }}$ is taken to be the maximum a posteriori (MAP) value. Note that Ref. [61] instead defined the recovered value to be the median value while Refs. [19,25] used the parameters that maximize the match, which is similar to the maximum likelihood estimate.

We now summarize to what extent our results are consistent with previous ones. Broadly speaking, our findings are in agreement with both Kalaghatgi et al. and Varma et al., although there are some differences, which is to be expected. Indeed, our injected signals have larger SNRs, our gravitational-wave recovery model is different, and our setup uses a coherent Bayesian inference on the combined datasets from the current three-detector network of observatories.

\section{Comparison to Varma et al.}

References [19,25] have used NR hybrids to map out where in the parameter space systematic errors from using quadrupole-only templates dominate over the expected $1 \sigma$ statistical errors. Such regions characterize where neglecting subdominant modes will lead to unacceptably large 
errors in the parameter estimates. Statistical errors were estimated using Fisher information matrix approximations with a single detector setup, while the value of $\lambda_{\text {recovered }}$ was taken to be the best fit parameter values using a IMRPhenonD recovery model. The injected signal's strength was set to achieve a sky-averaged value of $\mathrm{SNR}=8$ (corresponding to an optimal orientation SNR of about 20), and they take a weighted average of the bias over a population of binaries with isotropic orientations. Finally, while the effective spins of the injections they consider are similar to ours, the individual spin components are different.

Our main point of comparison is with Fig. 1 of Ref. [19], where the authors identify where in the parameter space subdominant modes are important by considering where $\beta_{\lambda}$ exceeds 1. By this measure, in our study subdominant modes are important for parameter estimation for all of the cases shown in Fig. 10 except $\chi_{\text {eff }}=0.5$ and $q \leq 4$. By comparison, Varma et al. find that nearly all of these cases show no bias; only $\chi_{\text {eff }}=-0.5$ and large-mass ratio systems are require subdominant modes to be included in the model. As such, for heavy BBH systems, our results indicate that subdominant modes are required over a larger region of the parameter space as compared to the general conclusions of Ref. [19]. The most likely explanation for this discrepancy is the different SNR values used in our studies. While typically the largest SNR in any given detector is about 20, our signal's network SNR is 30 .

We also point out that all of the trends evident in Fig. 1 of Ref. [19] have been confirmed in our fully Bayesian, threedetector setup. Most interestingly that at a fixed SNR the impact of subdominant modes will depend strongly on $\chi_{\text {eff }}$,
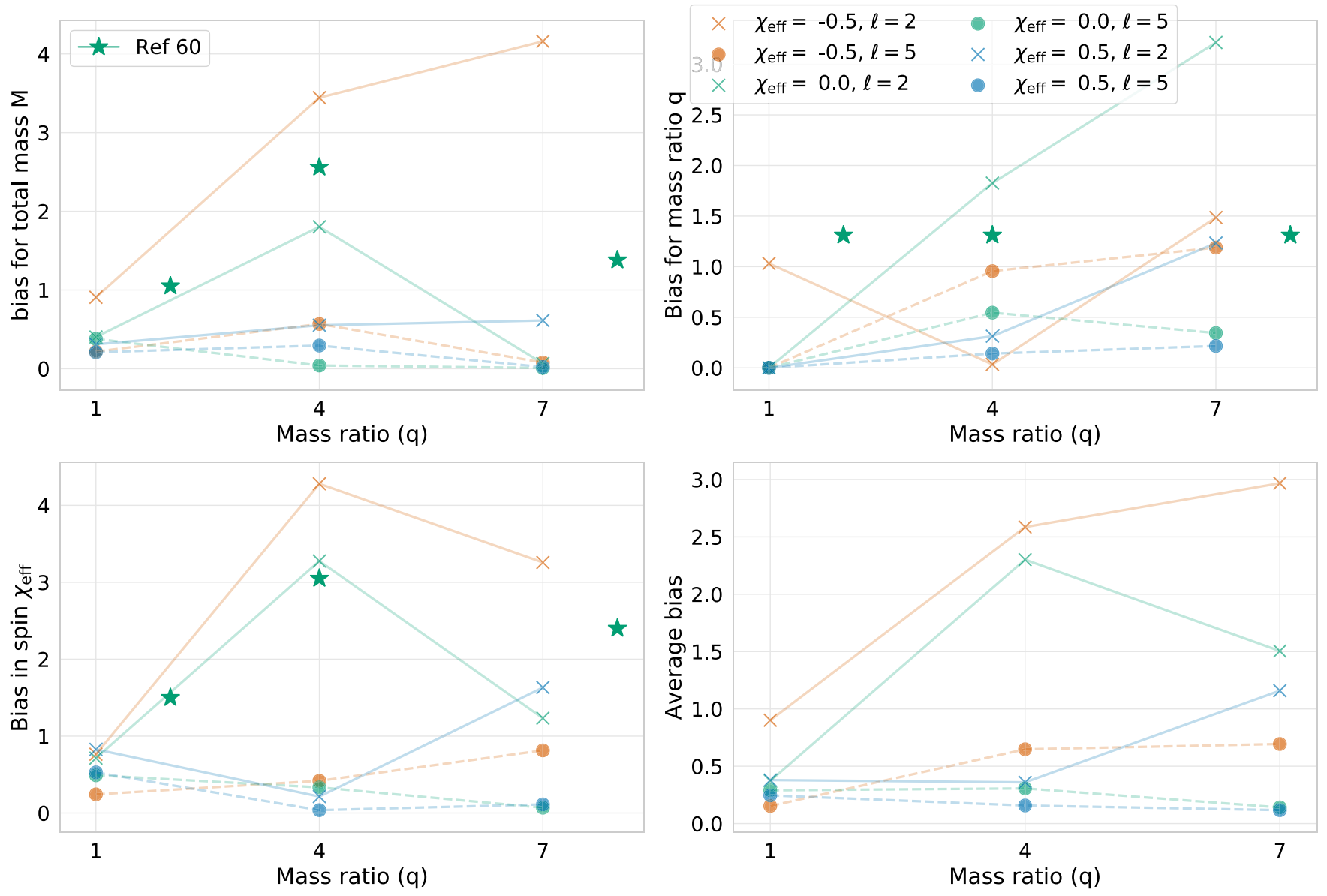

FIG. 10. Parameter biases, $\beta_{\lambda}$, for $\lambda=\left\{M, q, \chi_{\mathrm{e} f f}\right\}$ recovered with either all $\ell_{\max }=5$ modes (dashed line) or $\ell_{\text {max }}=2$ modes (solid line). We consider different synthetic sources by varying $q=\{1,4,7\}$ and $\chi_{\mathrm{e} f f}=\{-.5,0, .5\}$ while fixing SNR $=30$ and $M=120$. (Note: The marginalized posteriors for these systems are shown in Figs. 1, 2, and 3 which report the $90 \%$ confidence interval values, instead of the $68 \%$ values used to compute $\beta_{\lambda}$ ). To compare with Ref. [61], we also show the bias (green stars) for a similar set of nonspinning synthetic sources recovered with the quadrapole-only IMRPhenomD model. Broadly speaking, given the many differences in our setup, our findings are in general agreement with Ref. [61]. Note that the apparent disagreement in $\beta_{q}$ appears to be due to our definition of the bias (see text). Finally, we also show the average bias, $\left(\beta_{M}+\beta_{q}+\beta_{\chi_{\text {eff }}}\right) / 3$ in the bottom right panel. Here we clearly see general trends typically observed in our studies: subdominant modes are increasingly important as the value of the mass ratio increases and/or effective spin decreases, and recovery models that include all modes reduces bias in all cases. 
with almost no bias observed for large, positive spins. We return to this issue in the conclusions.

\section{Comparison to Kalaghatgi et al.}

A very recent study by Kalaghatgi et al. [61] used a two-detector Bayesian setup and studied the impact of subdominant modes for nonspinning systems while systematically varying the inclination angle. In this study, NR hybrids are used as the signal template and a quadrupoleonly IMRPhenomD recovery model is used. Indeed, their choice of $M=100$ and SNR $=25$ makes their setup closely analogous to ours, which facilitates direct comparison for non-spinning systems. We compare to their set of runs where the injected signal's inclination is set to 60 degrees, which is close to our value of 45 degrees.

In Fig. 10 we plot (green star) the bias due to omitting subdominant modes as reported in Ref. [61]. These should be compared with our nonspinning, $\ell_{\max }=2$ (green circles; solid green line) biases. The dependence of $\beta_{M}$ and $\beta_{\chi_{\text {eff }}}$ with mass ratio is in broad agreement, with both results showing a similar up-down pattern. Our smaller values of $\beta_{M}$ and $\beta_{\chi_{\text {eff }}}$ indicate less error due to neglecting subdominant modes, which is somewhat surprising seeing as our network SNR is larger. This is most likely due to the fact that we inject and recover with the same NR surrogate model. Our values for $\beta_{q}$ appear to show disagreement, which is mostly due to differing choices for the recovered value. Indeed, since many of our posteriors in $q$ peak at $q=1$ the bias is 0 , whereas the mean is offset from 1 . We have checked that when switching to the definition used in Kalaghatgi et al. our bias values are more consistent with values of about 1.4, 1.4 and 2.7 at $q=1, q=4$, and $q=7$, respectively.

\section{F. Measuring individual black hole spins}

It is well known that while individual spins are difficult to measure, the effective spin parameter, $\chi_{\text {eff }}$, is much better constrained. A recent study [69] systematically explored this question in the context of a single gravitational-wave detector by using the quadrapole-only SEOBNRv2 model $[70,71]$. The general conclusion of this work (see Figs. 1 and 4 of Ref. [69]) is that individual spins are poorly constrained. For equal-mass systems, it was found that the spin measurements are constrained only by the Kerr limit and so only near-extremal spins can be constrained as the posterior will run up against the prior. Furthermore, as the mass ratio increases, the spin of the larger blackhole is better constrained while the smaller black hole's spin remains unconstrained. Finally, this general picture remains unchanged across a wide range of total masses, including the values we have focused on in our paper.

In this subsection, we revisit the results from Sec. III but now briefly comment on our ability to measure the individual component spins using the full three-detector network with a our multimode recovery model.
Unfortunately, as anticipated in Ref. [69], the inclusion of subdominant modes does not qualitatively change the situation. This is visually and quantitatively evident for equal mass (cf. Fig. 1), $q=4$ (cf. Fig. 2), and $q=7$ (cf. Fig. 3) systems, all of which have a network SNR of 30. Here we see that while the inclusion of subdominant modes (dashed lines) dramatically reduces the bias in recovering $\chi_{\text {eff }}, \chi_{1}$, and $\chi_{2}$, the size of the $90 \%$ confidence intervals (shown in the figure's title) are mostly unaffected. A similar conclusion can be reached by comparing the joint distributions for $\chi_{1}$ vs $\chi_{2}$ (bottom right panels in Figs. 1, 2, and 3) recovered with $\ell_{\max }=2$ and $\ell_{\max }=5$ recovery models.

Thus we conclude that, at least for the configurations considered here, including subdominant modes in our waveform recovery model will reduce bias in the both the effective spin and individual spin components, but does relatively little to better constrain them.

\section{CONCLUSIONS}

In this work, using the recently-developed NRHybSur3dq8 model, we systematically investigate the importance of higher modes on the interpretation of gravitational wave signals from coalescing binary black hole systems. We have primarily focused on heavy systems with masses and spins similar to the detector-frame masses of near-future gravitational-wave observations while using current detector network sensitivities. Previous studies [19-27,30,32,61] have also explored this question in various approximate contexts, either using a single detector, relying on Fisher information matrix approximations, or restricted to non-spinning BBH models. Here we perform coherent Bayesian inference on the combined datasets from the current three-detector network of observatories, which is the same setup used in the recent analysis of gravitational wave observations [9]. We confirm many of the general expectations of previous works, while providing a more direct quantification of the bias within this realistic setup.

As expected, we find that higher modes are very important for interpreting asymmetric binaries with $q>1$. More surprisingly, we find noticeable differences even when the injected signal mass ratio is $q=1$, when subdominant modes are expected to be suppressed (See Appendix for a small follow up analysis). Also as expected, we find that the biases introduced by neglecting highermodes are very important for $q>1$ and $\mathrm{SNR} \geq 30$ $[19,26,59]$. However, in our examples we also find that inference without higher modes has a significant impact on the interpretation of low-SNR sources, particularly by influencing our knowledge of the binary's mass ratio. General trends typically observed in our studies indicate that subdominant modes are increasingly important as the value of the mass ratio increases and/or effective spin decreases, and recovery models that include all modes reduces bias in all cases. Our work highlights the 
importance of subdominant modes for events similar to GW190412, an unequal mass BBH merger.

Consistent with previous work, we find that configurations with $M_{z} \simeq 120$ and large aligned spins have almost no parameter bias [19] even at high SNRs. Such systems with large aligned spin exhibit the orbital hangup effect and have more in-band cycles. Given that the systems we have considered start in the late-inspiral regime, results from numerical relativity are most relevant toward quantifying the importance of this effect. For example, Table 3 from Ref. [72] shows that when starting from a fixed gravitational-wave frequency, the number of premerger orbits from an equal-mass, spin-aligned BBH system increases from about 5 to 9 as the effective spin parameter is varied from 0 to 0.85 . Figure 11 shows an example of this effect for the two most extreme cases we have considered in our study. The time-domain inset shows that the length of the signal increases as the spin becomes more positive, hence more of the SNR will be contained in the inspiral for systems with large, positive spin. The inspiral portion of the signal is known to be dominated by the $(2,2)$ mode's amplitude [20], which Fig. 11's insets show by comparing the relative amplitudes. We also see that near and after merger the higher modes quickly become larger in amplitude. Hence the impact of higher modes will be suppressed for longer signals, which seems to be why the orbital hangup effect serves to suppress the importance of higher modes. Other mechanisms by which more of the inspiral is in-band should similarly reduce the importance of higher harmonics. For instance, at a fixed SNR, the importance of subdominant modes for parameter estimation with systems with total masses lighter (heavier) than the fiducial value of 120 considered here are expected to be less (more) important for parameter estimation.

In our examples, parameter inference of spinning BBH systems without higher modes are frequently biased. These consistent systematic biases may accumulate in population inference calculations, as described in Sec. IV C (see also Ref. [68]). We anticipate that any population inferences of asymmetric, high-mass black hole binaries will require significant attention to waveform systematics. Section IV B also demonstrated that neglecting higher-order modes in the analysis of GW observations leads to biased estimates of the remnant object's mass and spin. For instance, Fig. 8 shows that higher-modes provide significantly better constraints on the remnant values, while the computed Jensen-Shannon divergence indicates a tension between the remnant mass and spin posteriors recovered by the $\ell_{\max }=2$ model and the true one (cf. Fig. 8) over a range of SNRs and mass ratios. As the remnant values feature prominently in IMR consistency tests of general relativity, our study suggests that neglecting higher-modes could incorrectly trigger failed tests of GR, for example when carrying out consistency tests between the strong-field merger and ringdown portions of the signal. Despite the many benefits enumerated here, unfortunately, subdominant modes do not appear to improve our ability to resolve individual spin components, but they can reduce bias in their recovered values.

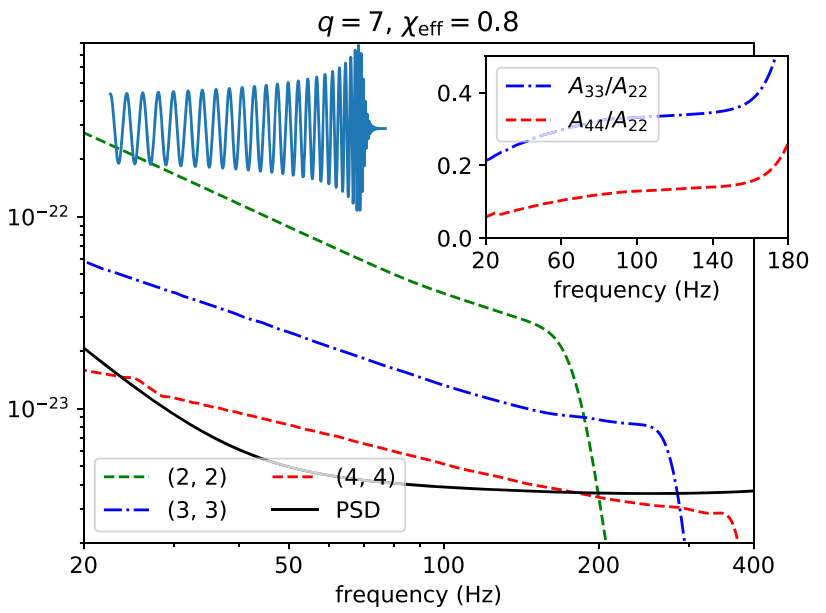

FIG. 11. These panels show the absolute value of the Fourier transform of the $(2,2),(3,3)$, and $(4,4)$ modes of the $q=7$ system with a spin of $\chi_{\text {eff }}=-0.8$ (left; ID12) and $\chi_{\text {eff }}=0.8$ (right; ID16), and for reference we show the ZeroDetHighPower PSD. The inset figures show the ratio of the higher modes relative to the dominant mode. Because the $\chi_{\text {eff }}=-0.8$ system merges at a lower orbital frequency, the subdominant modes contribute more to the overall SNR. This should be compared to the $\chi_{\text {eff }}=0.8$ system which merges at a higher orbital frequency, and consequently more of the inspiral portion of the waveform, which is dominated by the $(2,2)$ mode, contributes to the overall SNR. For illustrative purposes, a cartoon inset shows the time-domain signal starting from $20 \mathrm{~Hz}$ is of drastically different durations for these two systems. Note that the Fourier transformed signals were started from $3 \mathrm{~Hz}$ and tapered in order to avoid boundary effects. We also see that the waveform model has a small hybridization "glitch" in the $(4,4)$ mode, which is likely due to post-Newtonian theory breaking down at high mass ratio and high spin; hybridization will be improved when higher order PN amplitude terms become available. 
Finally, we have found that posteriors using an incomplete waveform model are often significantly offset from the full-model posterior, typically toward (incorrectly) favoring lighter binary systems with more negative $\chi_{\text {eff }}$ values. For example, a significant fraction of the probability for the $\ell_{\max }=5$ posterior is not contained within the high-probability boundaries of the $\ell_{\max }=2$ posterior. This suggests that it may be difficult to apply the likelihoodreweighting techniques advocated in [62], which require similar posterior distributions in all binary intrinsic and extrinsic parameters for the two models being applied (i.e., a simplified $\ell_{\max }=2$ model and a model including higher modes).

Given the large number of possible injection values one could consider, we have restricted our attention to systems with $M_{z}=120 M_{\odot}$ and $\chi_{1 z}=\chi_{2 z}$, while varying $\chi_{\text {eff }}, q$ and the SNR. By relaxing these restrictions, future studies should explore the importance of subdominant modes with coherent Bayesian inference using the three-detector network of observatories. Within a restricted setup, previous studies have shown that, generally speaking, the bias due to omitting subdominant modes increases at higher total masses $[19,20,25]$. Given that only the heaviest systems (e.g. GW170729) observed to date have a detector-frame total mass near $M_{z}=120 M_{\odot}$, our results provide a convenient upper bound on the greatest impact of subdominant modes for near-future binary black hole observations. A more comprehensive survey using our setup could be used to identify for which regions of the parameter space subdominant modes are important when considering total mass variations (cf. Fig. 1 of Ref. [19]).

Looking ahead, we anticipate that aligned-spin IMR models including higher modes [14-16] will become standard in the analysis gravitational wave observations. Indeed, as shown here, the inclusion of subdominant modes will improve the interpretation of most events, and in some cases substantially so. A very recent study by Kalaghatgi et al. [61], using $M_{z} \simeq 120 M_{\odot}$, nonspinning BBH systems and an aligned-spin phenomenological recovery model IMRPhenomHM, has also concluded that higher modes significantly reduces bias. Using the most physicallycomplete models will also remove the need for ad hoc regions-of-validity that depend on both the source parameters as well as the scientific questions under consideration. However, to enable our model to fully encompass the range of likely events, our models must also allow for generic precessing sources. Recent modeling of precessing binaries will allow for improved analysis of generic precessing sources [73]. Indeed, as already indicated by Ref. [37], we expect that many tests of general relativity could be biased unless they account for both higher modes and precession.

\section{ACKNOWLEDGMENTS}

We thank Gaurav Khanna for helpful discussions and providing technical assistance using the CARNiE cluster.
We thank Chinmay Kalaghatgi and Juan Calderon Bustillo for helpful comments on an earlier version of this manuscript, and the anonymous referee for numerous suggestions. R. O.S. and J. A. L. gratefully acknowledge NSF Award No. PHY-1707965. S. E. F. is partially supported by NSF Grant No. PHY-1806665, and F. H. S. is supported by NSF Grant No. PHY-1806665 and the UMassD Physics Department. L.E. K. acknowledges support from the Sherman Fairchild Foundation and NSF Grant No. PHY1606654 at Cornell. The computational work of this project was performed on the CARNiE cluster at UMassD, which is supported by the ONR/DURIP Grant No. N00014181255. S. E. F. and F. H. S. thank the Center for Scientific Computing \& Visualization Research (CSCVR) for both its technical support and for its hospitality while part of this work was completed.

\section{APPENDIX: FOLLOW UP ON THE SIGNIFICANCE OF HIGHER MODES FOR EQUAL MASS, ZERO SPIN, SNR = 10 CASE}

As pointed out in Secs. III D and V, there seems to be significant differences between the $\ell_{\max }=5$ and $\ell_{\max }=2$ runs for the equal mass, zero spin, $\mathrm{SNR}=10$ case, which runs contrary to several previous studies that had implied that HM would have minimal impact at low SNR for comparable-mass binaries.

To better understand our results, we perform a complementary analysis under the assumption of zero spin

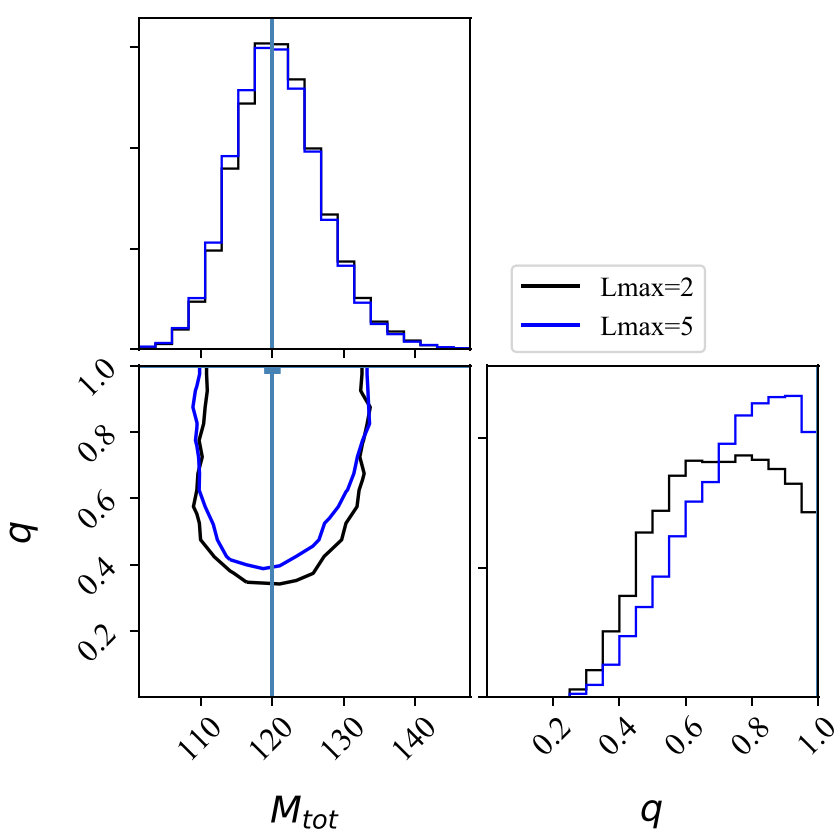

FIG. 12. Reanalysis of equal mass, zero spin, SNR = 10: This corner plot shows the reanalyses of a equal mass, zero spin, $\mathrm{SNR}=10$ source using $\ell_{\max }=2$ (black) and $\ell_{\max }=5$ (blue) mode but only on a grid in mass parameters (i.e., assuming zero spin). As first shown in Fig. 4, there are noticeable differences between the two different distributions. 
(i.e., lay out a grid only in $M_{\text {tot }}, q$ ), allowing us to directly evaluate the marginal likelihood versus the two remaining binary parameters. Figure 12 shows the results of both the $\ell_{\max }=5$ and $\ell_{\max }=2$ results. We continue to observe notable differences between the two posteriors even when restricted to two dimensions (i.e., only mass parameters). It is certainly surprising to see any difference given that this is a low SNR, equal mass event. One possibility is that due to the broadness of the posterior in mass ratio, a significant fraction of the posterior needs to be evaluated at values of $q \gtrsim 2$ where higher modes begin to play an increasingly important role.
[1] J. Aasi et al. (LIGO Scientific Collaboration), Advanced LIGO, Classical Quantum Gravity 32, 074001 (2015).

[2] T. Accadia et al. (VIRGO Collaboration), Virgo: A laser interferometer to detect gravitational waves, J. Instrum. 7, P03012 (2012).

[3] B. P. Abbott et al. (LIGO Scientific, Virgo Collaborations), Observation of Gravitational Waves from a Binary Black Hole Merger, Phys. Rev. Lett. 116, 061102 (2016).

[4] B. P. Abbott et al. (LIGO Scientific, Virgo Collaborations), Binary Black Hole Mergers in the first Advanced LIGO Observing Run, Phys. Rev. X 6, 041015 (2016); Erratum, Phys. Rev. X 8, 039903 (2018).

[5] B. P. Abbott et al. (LIGO Scientific, VIRGO Collaborations), GW170104: Observation of a 50-Solar-Mass Binary Black Hole Coalescence at Redshift 0.2, Phys. Rev. Lett. 118, 221101 (2017); Erratum, Phys. Rev. Lett. 121, 129901 (2018).

[6] B. P. Abbott et al. (LIGO Scientific, Virgo Collaborations), GW170814: A Three-Detector Observation of Gravitational Waves from a Binary Black Hole Coalescence, Phys. Rev. Lett. 119, 141101 (2017).

[7] B. P. Abbott et al. (LIGO Scientific, Virgo Collaborations), GW170608: Observation of a 19-solar-mass binary black hole coalescence, Astrophys. J. 851, L35 (2017).

[8] B. P. Abbott et al. (LIGO Scientific, Virgo Collaborations), GW170817: Observation of Gravitational Waves from a Binary Neutron Star Inspiral, Phys. Rev. Lett. 119, 161101 (2017).

[9] B. P. Abbott et al. (LIGO Scientific, Virgo Collaborations), GWTC-1: A Gravitational-Wave Transient Catalog of Compact Binary Mergers Observed by LIGO and Virgo during the First and Second Observing Runs, Phys. Rev. X 9, 031040 (2019).

[10] B. P. Abbott et al. (LIGO Scientific, Virgo Collaborations), Astrophysical implications of the binary black-hole merger GW150914, Astrophys. J. 818, L22 (2016).

[11] M. Hannam, P. Schmidt, A. Boh, L. Haegel, S. Husa, F. Ohme, G. Pratten, and M. Pürrer, Simple Model of Complete Precessing Black-Hole-Binary Gravitational Waveforms, Phys. Rev. Lett. 113, 151101 (2014).

[12] A. Taracchini et al., Effective-one-body model for blackhole binaries with generic mass ratios and spins, Phys. Rev. D 89, 061502 (2014).

[13] Y. Pan, A. Buonanno, A. Taracchini, L. E. Kidder, A. H. Mrou, H. P. Pfeiffer, M. A. Scheel, and B. Szilgyi, Inspiralmerger-ringdown waveforms of spinning, precessing blackhole binaries in the effective-one-body formalism, Phys. Rev. D 89, 084006 (2014).
[14] L. London, S. Khan, E. Fauchon-Jones, C. Garca, M. Hannam, S. Husa, X. Jimnez-Forteza, C. Kalaghatgi, F. Ohme, and F. Pannarale, First Higher-Multipole Model of Gravitational Waves from Spinning and Coalescing BlackHole Binaries, Phys. Rev. Lett. 120, 161102 (2018).

[15] R. Cotesta, A. Buonanno, A. Boh, A. Taracchini, I. Hinder, and S. Ossokine, Enriching the symphony of gravitational waves from binary black holes by tuning higher harmonics, Phys. Rev. D 98, 084028 (2018).

[16] V. Varma, S. E. Field, M. A. Scheel, J. Blackman, L. E. Kidder, and H. P. Pfeiffer, Surrogate model of hybridized numerical relativity binary black hole waveforms, Phys. Rev. D 99, 064045 (2019).

[17] J. Blackman, S. E. Field, M. A. Scheel, C. R. Galley, D. A. Hemberger, P. Schmidt, and R. Smith, A surrogate model of gravitational waveforms from numerical relativity simulations of precessing binary black hole mergers, Phys. Rev. D 95, 104023 (2017).

[18] J. Blackman, S. E. Field, M. A. Scheel, C. R. Galley, C. D. Ott, M. Boyle, L. E. Kidder, H. P. Pfeiffer, and B. Szilgyi, Numerical relativity waveform surrogate model for generically precessing binary black hole mergers, Phys. Rev. D 96, 024058 (2017).

[19] V. Varma and P. Ajith, Effects of nonquadrupole modes in the detection and parameter estimation of black hole binaries with nonprecessing spins, Phys. Rev. D 96, 124024 (2017).

[20] J. C. Bustillo, S. Husa, A. M. Sintes, and M. Pürrer, Impact of gravitational radiation higher order modes on single aligned-spin gravitational wave searches for binary black holes, Phys. Rev. D 93, 084019 (2016).

[21] C. Capano, Y. Pan, and A. Buonanno, Impact of higher harmonics in searching for gravitational waves from nonspinning binary black holes, Phys. Rev. D 89, 102003 (2014).

[22] T. B. Littenberg, J. G. Baker, A. Buonanno, and B. J. Kelly, Systematic biases in parameter estimation of binary blackhole mergers, Phys. Rev. D 87, 104003 (2013).

[23] J. C. Bustillo, P. Laguna, and D. Shoemaker, Detectability of gravitational waves from binary black holes: Impact of precession and higher modes, Phys. Rev. D 95, 104038 (2017).

[24] D. A. Brown, P. Kumar, and A. H. Nitz, Template banks to search for low-mass binary black holes in advanced gravitational-wave detectors, Phys. Rev. D 87, 082004 (2013).

[25] V. Varma, P. Ajith, S. Husa, J. C. Bustillo, M. Hannam, and M. Pürrer, Gravitational-wave observations of binary black 
holes: Effect of nonquadrupole modes, Phys. Rev. D 90, 124004 (2014).

[26] P. B. Graff, A. Buonanno, and B. S. Sathyaprakash, Missing Link: Bayesian detection and measurement of intermediatemass black-hole binaries, Phys. Rev. D 92, 022002 (2015).

[27] I. Harry, J. C. Bustillo, and A. Nitz, Searching for the full symphony of black hole binary mergers, Phys. Rev. D 97, 023004 (2018).

[28] R. O'Shaughnessy, B. Farr, E. Ochsner, H.-S. Cho, V. Raymond, C. Kim, and C.-H. Lee, Parameter estimation of gravitational waves from precessing black hole-neutron star inspirals with higher harmonics, Phys. Rev. D 89, 102005 (2014).

[29] S. A. Usman, J. C. Mills, and S. Fairhurst, Constraining the inclinations of binary mergers from gravitational-wave observations, Astrophys. J. 877, 82 (2019).

[30] P. Kumar, J. Blackman, S. E. Field, M. Scheel, C. R. Galley, M. Boyle, L. E. Kidder, H. P. Pfeiffer, B. Szilagyi, and S. A. Teukolsky, Constraining the parameters of GW150914 and GW170104 with numerical relativity surrogates, Phys. Rev. D 99, 124005 (2019).

[31] R. Abbott et al. (LIGO Scientific, Virgo Collaborations), GW190412: Observation of a binary-black-hole coalescence with asymmetric masses, arXiv:2004.08342.

[32] B. P. Abbott et al. (LIGO Scientific, Virgo Collaborations), Effects of waveform model systematics on the interpretation of GW150914, Classical Quantum Gravity 34, 104002 (2017).

[33] B. P. Abbott et al. (LIGO Scientific, Virgo Collaborations), Directly comparing GW150914 with numerical solutions of Einsteins equations for binary black hole coalescence, Phys. Rev. D 94, 064035 (2016).

[34] K. Chatziioannou et al., On the properties of the massive binary black hole merger GW170729, Phys. Rev. D 100, 104015 (2019).

[35] B. P. Abbott et al. (LIGO Scientific Collaboration, Virgo Collaboration), Prospects for observing and localizing gravitational-wave transients with advanced ligo and advanced virgo, Living Rev. Relativity 19, 1 (2016).

[36] J. C. Bustillo, J. A. Clark, P. Laguna, and D. Shoemaker, Tracking Black Hole Kicks from Gravitational Wave Observations, Phys. Rev. Lett. 121, 191102 (2018).

[37] P. T. H. Pang, J. C. Bustillo, Y. Wang, and T. G. F. Li, Potential observations of false deviations from general relativity in gravitational wave signals from binary black holes, Phys. Rev. D 98, 024019 (2018).

[38] K. Belczynski et al., The effect of pair-instability mass loss on black hole mergers, Astron. Astrophys. 594, A97 (2016).

[39] B. McKernan, K. E. S. Ford, R. O'Shaughnessy, and D. Wysocki, Monte-Carlo simulations of black hole mergers in AGN disks: Low $\chi_{\text {eff }}$ mergers and predictions for LIGO, Mon. Not. R. Astron. Soc. 494, 1203 (2020).

[40] T. Damour, Coalescence of two spinning black holes: An effective one-body approach, Phys. Rev. D 64, 124013 (2001).

[41] E. Racine, Analysis of spin precession in binary black hole systems including quadrupole-monopole interaction, Phys. Rev. D 78, 044021 (2008).

[42] P. Ajith et al., Inspiral-Merger-Ringdown Waveforms for Black-Hole Binaries with Non-Precessing Spins, Phys. Rev. Lett. 106, 241101 (2011).
[43] LIGO Scientific Collaboration, LIGO Algorithm LibraryLALSuite, free software (GPL) (2018).

[44] GWSurrogate, https://pypi.python.org/pypi/gwsurrogate/.

[45] S. E. Field, C. R. Galley, J. S. Hesthaven, J. Kaye, and M. Tiglio, Fast prediction and evaluation of gravitational waveforms using surrogate models, Phys. Rev. X 4, 031006 (2014).

[46] E. E. Flanagan and S. A. Hughes, Measuring gravitational waves from binary black hole coalescences: 2. The Waves' information and its extraction, with and without templates, Phys. Rev. D 57, 4566 (1998).

[47] L. Lindblom, B. J. Owen, and D. A. Brown, Model waveform accuracy standards for gravitational wave data analysis, Phys. Rev. D 78, 124020 (2008).

[48] S. T. McWilliams, B. J. Kelly, and J. G. Baker, Observing mergers of non-spinning black-hole binaries, Phys. Rev. D 82, 024014 (2010).

[49] K. Chatziioannou, A. Klein, N. Cornish, and N. Yunes, Analytic Gravitational Waveforms for Generic Precessing Binary Inspirals, Phys. Rev. Lett. 118, 051101 (2017).

[50] E. Baird, S. Fairhurst, M. Hannam, and P. Murphy, Degeneracy between mass and spin in black-hole-binary waveforms, Phys. Rev. D 87, 024035 (2013).

[51] M. Boyle et al., The SXS Collaboration catalog of binary black hole simulations, Classical Quantum Gravity 36, 195006 (2019).

[52] M. Vallisneri and N. Yunes, Stealth bias in gravitationalwave parameter estimation, Phys. Rev. D 87, 102002 (2013).

[53] A. R. Williamson, J. Lange, R. O'Shaughnessy, J. A. Clark, P. Kumar, J. C. Bustillo, and J. Veitch, Systematic challenges for future gravitational wave measurements of precessing binary black holes, Phys. Rev. D 96, 124041 (2017).

[54] M. Favata, Systematic Parameter Errors in Inspiraling Neutron Star Binaries, Phys. Rev. Lett. 112, 101101 (2014).

[55] J. Veitch et al., Parameter estimation for compact binaries with ground-based gravitational-wave observations using the LALInference software library, Phys. Rev. D 91, 042003 (2015).

[56] J. Lange, R. O'Shaughnessy, and M. Rizzo, Rapid and accurate parameter inference for coalescing, precessing compact binaries, arXiv:1805.10457.

[57] C. Pankow, P. Brady, E. Ochsner, and R. O’Shaughnessy, Novel scheme for rapid parallel parameter estimation of gravitational waves from compact binary coalescences, Phys. Rev. D 92, 023002 (2015).

[58] J. Lange et al., Parameter estimation method that directly compares gravitational wave observations to numerical relativity, Phys. Rev. D 96, 104041 (2017).

[59] R. O'Shaughnessy, J. Blackman, and S. E. Field, An architecture for efficient gravitational wave parameter estimation with multimodal linear surrogate models, Classical Quantum Gravity 34, 144002 (2017).

[60] D. Wysocki, R. O'Shaughnessy, J. Lange, and Y.-L. L. Fang, Accelerating parameter inference with graphics processing units, Phys. Rev. D 99, 084026 (2019).

[61] C. Kalaghatgi, M. Hannam, and V. Raymond, Parameter Estimation with a spinning multi-mode waveform model: IMRPhenomHM, Phys. Rev. D 101, 103004 (2020). 
[62] E. Payne, C. Talbot, and E. Thrane, Higher order gravitational-wave modes with likelihood reweighting, Phys. Rev. D 100, 123017 (2019).

[63] M. Campanelli, C. O. Lousto, and Y. Zlochower, Spinningblack-hole binaries: The orbital hang up, Phys. Rev. D 74, 041501 (2006).

[64] K. Chatziioannou, G. Lovelace, M. Boyle, M. Giesler, D. A. Hemberger, R. Katebi, L. E. Kidder, H. P. Pfeiffer, M. A. Scheel, and B. Szilágyi, Measuring the properties of nearly extremal black holes with gravitational waves, Phys. Rev. D 98, 044028 (2018).

[65] B. P. Abbott et al. (LIGO Scientific, Virgo Collaborations), Tests of General Relativity with GW150914, Phys. Rev. Lett. 116, 221101 (2016); Erratum, Phys. Rev. Lett. 121, 129902 (2018).

[66] I. Ota and C. Chirenti, Overtones or higher harmonics? Prospects for testing the no-hair theorem with gravitational wave detections, Phys. Rev. D 101, 104005 (2020).

[67] V. Varma, D. Gerosa, L. C. Stein, F. Hbert, and H. Zhang, High-Accuracy Mass, Spin, and Recoil Predictions of Generic Black-Hole Merger Remnants, Phys. Rev. Lett. 122, 011101 (2019).

[68] D. Wysocki, J. Lange, and R. O'Shaughnessy, Reconstructing phenomenological distributions of compact binaries via gravitational wave observations, Phys. Rev. D 100, 043012 (2019).

[69] M. Pürrer, M. Hannam, and F. Ohme, Can we measure individual black-hole spins from gravitational-wave observations?, Phys. Rev. D 93, 084042 (2016).

[70] M. Pürrer, Frequency-domain reduced order models for gravitational waves from aligned-spin compact binaries, Classical Quantum Gravity 31, 195010 (2014).

[71] A. Taracchini, A. Buonanno, Y. Pan, T. Hinderer, M. Boyle, D. A. Hemberger, L. E. Kidder, G. Lovelace, A. H. Mroue, H. P. Pfeiffer, M. A. Scheel, B. Szilágyi, N. W. Taylor, and A. Zenginoglu, Effective-one-body model for black-hole binaries with generic mass ratios and spins, Phys. Rev. D 89, 061502(R) (2014).

[72] M. Hannam, S. Husa, B. Brügmann, and A. Gopakumar, Comparison between numerical-relativity and postNewtonian waveforms from spinning binaries: The orbital hang-up case, Phys. Rev. D 78, 104007 (2008).

[73] V. Varma, S. E. Field, M. A. Scheel, J. Blackman, D. Gerosa, L. C. Stein, L. E. Kidder, and H. P. Pfeiffer, Surrogate models for precessing binary black hole simulations with unequal masses, Phys. Rev. Research 1, 033015 (2019). 